\title{
Nonsingularity and Stationarity Results for Quasi-Variational Inequalities
}

\author{
Axel Dreves $^{1}$ (D) S Simone Sagratella ${ }^{2}$
}

Received: 23 September 2019 / Accepted: 27 April 2020 / Published online: 27 May 2020

(c) The Author(s) 2020

\begin{abstract}
The optimality system of a quasi-variational inequality can be reformulated as a nonsmooth equation or a constrained equation with a smooth function. Both reformulations can be exploited by algorithms, and their convergence to solutions usually relies on the nonsingularity of the Jacobian, or the fact that the merit function has no nonoptimal stationary points. We prove new sufficient conditions for the absence of nonoptimal constrained or unconstrained stationary points that are weaker than some known ones. All these conditions exploit some properties of a certain matrix, but do not require the nonsingularity of the Jacobian. Further, we present new necessary and sufficient conditions for the nonsingularity of the Jacobian that are based on the signs of certain determinants. Additionally, we consider generalized Nash equilibrium problems that are a special class of quasi-variational inequalities. Exploiting their structure, we also prove some new sufficient conditions for stationarity and nonsingularity results.
\end{abstract}

Keywords Quasi-variational inequality · Generalized Nash equilibrium problem • Nonsingularity $\cdot$ Nonoptimal stationary points

Mathematics Subject Classification 65K10 - 90C33

Communicated by Roland Herzog.

$凶 \quad$ Axel Dreves

axel.dreves@unibw.de

Simone Sagratella

sagratella@diag.uniroma1.it

1 Department of Aerospace Engineering, University of the Bundeswehr Munich,

Werner-Heisenberg-Weg 39, 85577 Neubiberg, Germany

2 Department of Computer, Control, and Management Engineering Antonio Ruberti, Sapienza University of Rome, Via Ariosto 25, 00185 Rome, Italy 


\section{Introduction}

We consider quasi-variational inequalities (QVIs). This problem class was introduced in the paper series [1-3]. It generalizes the classical variational inequality by allowing dependence of the feasible set from the point under consideration. We will define the problem at the beginning of the next section. For a detailed discussion of QVIs, we refer to $[4,5]$. Here, we will discuss the central conditions to ensure that general algorithms for QVIs are well defined and converge. These are conditions guaranteeing that stationary points of a merit function are indeed solutions, and conditions guaranteeing nonsingularity of some Jacobian matrix in order to compute the descent direction. Generalized Nash equilibrium problems (GNEPs) that can be used, for example, to model markets with several players sharing common constraints and that were first formally introduced in [6] can be reformulated as QVIs under mild conditions, see [7], and are hence one of the applications of QVIs. There are a growing number of GNEP applications, and we refer to the appendix of [8] for a test library and references. Further applications can be found, for example, in biological [9], mechanical [4,5], economic [10,11] or transportation problems [12,13]. Since several algorithms for GNEPs have been introduced in the recent years, we also discuss some of our issues for special cases of GNEPs, which are typically in a subclass of QVIs that is not easy to solve in practice.

Algorithms for QVIs or GNEPs have to deal with the fact that solutions are typically nonunique and often nonisolated, resulting in infinitely many solutions and singularity of the Jacobian matrix at the solutions, which in turn make the application of classical Newton methods difficult. Thus, the numerical solution of these problems is mathematically challenging. To prove uniqueness of the solution one usually has restrictive assumptions; see, for example, [14,15]. However, to get well-defined algorithms one usually only requires nonsingularity at the iterates, and hence, interior point methods are promising approaches since they restrict the iterates to a certain set. For general Newton methods, nonsingularity at the solution is often exploited to get superlinear local convergence. In the case of nonisolated solutions, which we typically have for QVIs, one can still construct local fast convergent algorithms by exploiting some error bound condition. This has been done in the GNEP setting in [16,17].

A promising approach to solve QVIs is to characterize the corresponding KarushKuhn-Tucker (KKT) points as zeros of a nonsmooth function. Then, one can apply a semismooth Newton method, as it was done in [18]. For the semismooth Newton approach in the context of GNEPs, see also [19]. For well-defined local methods, one requires nonsingularity of an element of the generalized Jacobian of the nonsmooth function at an iterate. For global convergence, one can use a linesearch procedure that is based on the squared norm of the nonsmooth function as a merit function. A convergence result for semismooth Newton methods usually proves that accumulation points are stationary points of the merit function. Hence, results guaranteeing that stationary points of the merit function are indeed KKT points are required. Also, if one uses a Levenberg-Marquardt method, see, for example, [20], to overcome the nonsingularity problems, one minimizes the squared norm of the merit function and relies on stationarity results. 
A different approach, also based on the solution of the KKT conditions, is an interior point method, where one solves a constrained equation with a smooth function. A potential reduction algorithm for QVIs or GNEPs is presented in [21] and [19], respectively. This algorithm requires very similar nonsingularity properties as the semismooth Newton approach at all iterates, which lie in the interior of some suitable set here.

A further approach is a trust region method for constrained equations as introduced in $[22,23]$. This can be applied directly to the smooth constrained equation reformulation of the KKT system, which was done for GNEPs in [19,24]. At each iteration, one has to solve a linear equation system, which is the same as for the interior point approach, and one gets convergence to stationary points of the merit function. Hence, also in this approach nonsingularity and nonoptimal stationarity are the main issues.

Moreover, let us mention that there is an approach based on the KKT conditions, namely the LP-Newton method proposed in [25] that avoids the singularity issues by solving a linear program instead of a linear equation system. For this approach, there is also a globalization technique through a suitable nondifferentiable merit function given in [26]. This avoids the problem to accumulate at possibly nonoptimal stationary points of the merit function at least for (piecewise) smooth problems. The price one has to pay is that each iteration becomes more expensive, since one has to solve a linear program instead of a linear equation system at each iteration. Even these methods converge to stationary points of some constrained equation reformulations of the KKT conditions of the QVI, and it is fundamental to have conditions guaranteeing that these points are actual solutions.

For all these methods, the results of this paper are directly applicable and are the theoretical base of convergence to a solution of the QVI.

For a short overview on methods to solve QVIs, not only via their KKT conditions, we refer to the introduction in [21] and the references cited there and to [27,28]. Finally, there are some newer augmented Lagrangian or multiplier-penalty methods to solve QVIs proposed in [29,30] that are built on the sequential solution of simpler variational inequalities, as first proposed in [31].

In our paper, we consider the constrained equation reformulation of the KKT conditions of the QVI and the nonsmooth unconstrained reformulation. We will present several conditions that are necessary and sufficient for the nonsingularity required by many of the algorithms mentioned above. In the context of QVIs, these are, to the best of our knowledge, the first necessary and sufficient conditions for nonsingularity of the Jacobians. Furthermore, we will present sufficient conditions that avoid the existence of nonoptimal stationary points for different constrained or unconstrained stationarity concepts. For the constrained reformulation, this is, again to the best of our knowledge, the first time that such kind of conditions are given in the QVI context. Furthermore, through our analysis we obtain hints about which specific KKT reformulation is preferable to be used with projected gradient-type methods or with interior point methods. We also extend existing results for QVIs and GNEPs from [18,21,32]. In particular, in [21] one can find several nonsingularity results for a number of special structured QVIs.

The paper is organized as follows: In Sect. 2, we introduce the problem formulation and define the relevant functions and matrices. Further, we present our assumptions 
and we state the main facts we want to prove in this paper: Figs. 1 and 2 anticipate and summarize the results proved in Sects. 3 and 4.

In Sect. 3, we derive new unconstrained and constrained stationarity results for QVIs and show their applicability in some examples: In Proposition 3.1, we generalize [19, Theorem 3.1] about unconstrained stationarity; in Proposition 3.2 and 3.3 we give conditions to get unconstrained stationarity results, and in Example 3.1 we show that without these conditions the stationarity results do not hold; in Theorem 3.1 and 3.2 we give new assumptions to get constrained stationarity results; Example 3.2 shows a QVI satisfying all assumptions of Theorem 3.2, while Example 3.3 and 3.4 show that without the conditions of Theorem 3.2 the constrained stationarity results may not hold.

Thereafter, in Sect. 4, we present nonsingularity results for QVIs and the main theorem provides a new necessary and sufficient condition: In Proposition 4.1, we state the equivalence between the nonsingularity of the Jacobians of the smooth and the nonsmooth reformulations; in Proposition 4.2 and 4.3 we report some technical conditions for nonsingularity results; in Proposition 4.4 we give sufficient conditions for nonsingularity results; our main Theorem 4.1 gives new necessary and sufficient conditions for the nonsingularity of the Jacobian of the smooth reformulation in the case of linear constraints with variable right-hand side; in the same framework, Corollary 4.1 shows that the conditions given in [21, Corollary 2] are not only sufficient, but also necessary, for the nonsingularity of the Jacobian of the smooth reformulation, while Corollary 4.2 and 4.3 state similar nonsingularity results for the nonsmooth reformulation.

We consider the special case of QVIs with box constraints in Sect. 5 and show that box constraints are useful to weaken our assumptions: Proposition 5.1 provides sufficient conditions to guarantee all assumptions of Proposition 3.2 and 3.3; Theorem 5.1 weakens the conditions of Theorem 3.2 for constrained stationarity results of the smooth reformulation; Corollary 5.1 and 5.2 deal with the nonsingularity issue by assuming less restrictive conditions.

Section 6 contains nonsingularity and stationarity results for GNEPs that are not straightforward applications of the corresponding QVI results, but exploit the special structure of GNEPs: Corollary 6.1 significantly improves [32, Theorem 4.1] for linear problems; Lemma 6.1 defines a class of GNEPs for which many nonsingularity and stationarity results hold, while Example 6.2 shows an application satisfying the conditions given in Lemma 6.1; in Lemma 6.2 we identify some GNEPs with box constraints that have nonsingularity and stationarity properties; Proposition 6.1 provides conditions for a GNEP with box constraints in order to obtain constrained stationarity properties for the smooth reformulation.

We close the paper with some final remarks in conclusions section.

\section{Problem Definition, Assumptions and Motivation}

We consider the following QVI: Find a vector $x^{*} \in K\left(x^{*}\right)$ such that

$$
F\left(x^{*}\right)^{\top}\left(y-x^{*}\right) \geq 0 \quad \forall y \in K\left(x^{*}\right)
$$


where $F: \mathbb{R}^{n} \rightarrow \mathbb{R}^{n}$ is a (point-to-point) mapping and $K: \mathbb{R}^{n} \rightrightarrows \mathbb{R}^{n}$ is a pointto-set mapping with closed and convex images. We assume that $F$ is continuously differentiable and that the feasible set mapping $K$ is given by a parametric set of inequality constraints:

$$
K(x):=\left\{y \in \mathbb{R}^{n}: g(y, x) \leq 0\right\},
$$

where $g: \mathbb{R}^{n} \times \mathbb{R}^{n} \rightarrow \mathbb{R}^{m}$ is twice continuously differentiable and, for each $i=1, \ldots, m, g_{i}(\cdot, x)$ is convex on $\mathbb{R}^{n}$, for each $x \in \mathbb{R}^{n}$. We could also include linear equations, which lead to additional smooth equations in the following optimality systems, but for simplicity of notation we drop them from our subsequent analysis. We set

$$
h(x):=g(x, x),
$$

and hence, $h(x) \leq 0$ means that $x \in K(x)$. We say that a point $x \in \mathbb{R}^{n}$ satisfies the KKT conditions, if multipliers $\lambda \in \mathbb{R}^{m}$ exist such that

$$
\begin{aligned}
& L(x, \lambda):=F(x)+\nabla_{y} g(x, x) \lambda=0 \\
& \lambda_{i} \geq 0, g_{i}(x, x) \leq 0, \lambda_{i} g_{i}(x, x)=0 \quad \forall i=1, \ldots, m .
\end{aligned}
$$

Note that $\nabla_{y} g(x, x)$ is the partial transposed Jacobian of $g(y, x)$ with respect to $y$ evaluated at $y=x$.

Our aim is to find a solution $x^{*}$ of the QVI by solving the KKT conditions (1). It is known, see, for example, [21, Theorem 1], that under the above convexity and differentiability assumptions, KKT points are solutions of the QVI. Further, under a suitable constraint qualification, like the Slater condition, all solutions of the QVI are KKT points. We can use the Fischer-Burmeister function as a complementarity function and define

$$
\varphi: \mathbb{R}^{m} \times \mathbb{R}^{m} \rightarrow \mathbb{R}^{m}, \quad \varphi(a, b):=\left(\begin{array}{c}
\sqrt{a_{1}^{2}+b_{1}^{2}}-a_{1}-b_{1} \\
\vdots \\
\sqrt{a_{m}^{2}+b_{m}^{2}}-a_{m}-b_{m}
\end{array}\right)
$$

to obtain: $\left(x^{*}, \lambda^{*}\right)$ is a KKT point of the QVI, if and only if $\left(x^{*}, \lambda^{*}\right)$ solves the nonlinear equation system

$$
0=\Psi(x, \lambda):=\left(\begin{array}{c}
L(x, \lambda) \\
\varphi(\lambda,-h(x))
\end{array}\right)
$$

Equivalently, $\left(x^{*}, \lambda^{*}\right)$ is a KKT point of the QVI, if and only if $\left(x^{*}, \lambda^{*}, w^{*}\right)$, with $w^{*}=-h\left(x^{*}\right)$, is a solution of the nonlinear constrained equation system

$$
0=H(x, \lambda, w):=\left(\begin{array}{c}
L(x, \lambda) \\
h(x)+w \\
\lambda \circ w
\end{array}\right) \quad(x, \lambda, w) \in \mathbb{R}^{n} \times \mathbb{R}_{+}^{m} \times \mathbb{R}_{+}^{m}
$$


Herein, $\lambda \circ w$ is the vector with the componentwise product, and $\mathbb{R}_{+}^{m}$ is the set of vectors with nonnegative components. Later on, we will also use $\mathbb{R}_{++}^{m}$ for the set of vectors with positive components. If $F$ is continuously differentiable and $g$ is twice continuously differentiable, it is well known that the Jacobian $J H$ of $H$ is given by

$$
J H(x, \lambda, w)=\left(\begin{array}{ccc}
J_{x} L(x, \lambda) & \nabla_{y} g(x, x) & 0 \\
J h(x) & 0 & I \\
0 & \operatorname{diag}(w) & \operatorname{diag}(\lambda)
\end{array}\right)
$$

see, for example, $[18,19,21]$. Here, $J_{x} L(x, \lambda)$ denotes the partial Jacobian of $L(x, \lambda)$ with respect to $x$, and $J h(x)$ is the Jacobian of $h$. Further, it is also known, see, for example, [19], that the generalized Jacobian of $\Psi$ is given by

$$
\partial \Psi(x, \lambda)=\left(\begin{array}{cc}
J_{x} L(x, \lambda) & \nabla_{y} g(x, x) \\
-D_{h} J h(x) & D_{\lambda}
\end{array}\right)
$$

where the matrices $D_{h}$ and $D_{\lambda}$ are $m \times m$ diagonal matrices, with entries given by

$$
\left(D_{h}^{i}, D_{\lambda}^{i}\right) \begin{cases}=\frac{\left(-h_{i}(x), \lambda_{i}\right)}{\sqrt{h_{i}(x)^{2}+\lambda_{i}^{2}}}-(1,1), & \text { if }\left(-h_{i}(x), \lambda_{i}\right) \neq(0,0), \\ \in \mathbb{B}_{1}-(1,1), & \text { if }\left(-h_{i}(x), \lambda_{i}\right)=(0,0),\end{cases}
$$

for all $i=1, \ldots, m$, and $\mathbb{B}_{1}$ denotes the closed unit ball in $\mathbb{R}^{2}$.

If the matrix $J_{x} L(x, \lambda)$ is nonsingular, we can define the reduced matrix

$$
M(x, \lambda):=\operatorname{Jh}(x) J_{x} L(x, \lambda)^{-1} \nabla_{y} g(x, x)
$$

that is related to both Jacobian matrices described above. We will use the notation $M_{i}$. to denote the $i$ th row of a matrix $M$. Let us recall some known matrix properties we will use, cf. [33].

- A matrix $M \in \mathbb{R}^{n \times n}$ is called a $P_{0}$ matrix, if for all $x \in \mathbb{R}^{n} \backslash\{0\}$ there is an index $i \in\{1, \ldots, n\}$ such that $x_{i} \neq 0$ and $x_{i} M_{i} \bullet x \geq 0$.

A matrix $M \in \mathbb{R}^{n \times n}$ is a $P_{0}$ matrix if all its principal minors are nonnegative.

- A matrix $M \in \mathbb{R}^{n \times n}$ is called a $P$ matrix, if for all $x \in \mathbb{R}^{n} \backslash\{0\}$ there is an index $i \in\{1, \ldots, n\}$ such that $x_{i} M_{i \bullet} x>0$.

A matrix $M \in \mathbb{R}^{n \times n}$ is a $P$ matrix if all its principal minors are positive.

- A matrix $M \in \mathbb{R}^{n \times n}$ is called a semimonotone matrix, if for all $x \in \mathbb{R}_{+}^{n} \backslash\{0\}$ there is an index $i \in\{1, \ldots, n\}$ such that $x_{i}>0$ and $M_{i \bullet} x \geq 0$.

Every $P_{0}$ matrix is semimonotone.

Every nonnegative matrix is semimonotone.

The sum of a semimonotone matrix and a nonnegative one is semimonotone.

- A matrix $M \in \mathbb{R}^{n \times n}$ is an $S$ matrix if $x \in \mathbb{R}_{++}^{n}$ exists such that $M x>0$.

An important practical issue in the design of algorithms consists in understanding if all stationary points of the merit function $\frac{1}{2}\|H(x, \lambda, w)\|^{2}$, with 
$(x, \lambda, w) \in \mathbb{R}^{n} \times \mathbb{R}_{+}^{m} \times \mathbb{R}_{+}^{m}$, and of the merit function $\frac{1}{2}\|\Psi(x, \lambda)\|^{2}$, possibly with $(x, \lambda) \in \mathbb{R}^{n} \times \mathbb{R}_{+}^{m}$, are zeros of $H$ and $\Psi$, respectively.

Moreover, the main topic in order to guarantee that interior point algorithms are well defined and converge to a solution of the QVI is the nonsingularity of $J H(x, \lambda, w)$ for $(x, \lambda, w) \in \mathbb{R}^{n} \times \mathbb{R}_{++}^{m} \times \mathbb{R}_{++}^{m}$ or of the elements of $\partial \Psi(x, \lambda)$ for $(x, \lambda) \in \mathbb{R}^{n} \times \mathbb{R}_{++}^{m}$. Note that for $\lambda \in \mathbb{R}_{++}^{m}$ the set $\partial \Psi(x, \lambda)$ is by definition single valued and contains only the Jacobian $J \Psi(x, \lambda)$.

More specifically, and summarizing, we are interested in giving conditions guaranteeing the following facts:

(F1) all stationary points of the merit function $\frac{1}{2}\|\Psi(x, \lambda)\|^{2}$ are zeros of $\Psi$;

(F2) all stationary points of the merit function $\frac{1}{2}\|H(x, \lambda, w)\|^{2}$, with $\lambda \geq 0$ and $w \geq 0$, are zeros of $H$

(F3) all stationary points of the merit function $\frac{1}{2}\|\Psi(x, \lambda)\|^{2}$, with $\lambda \geq 0$ and $h(x) \leq 0$, are zeros of $\Psi$

(F4) all constrained stationary points of

$$
\min \frac{1}{2}\|\Psi(x, \lambda)\|^{2} \quad \text { s.t. } \quad \lambda \geq 0
$$

are zeros of $\Psi$;

(F5) all constrained stationary points of

$$
\min \frac{1}{2}\|H(x, \lambda, w)\|^{2} \quad \text { s.t. } \quad \lambda \geq 0, w \geq 0
$$

are zeros of $H$;

(F6) $J H(x, \lambda, w)$ is nonsingular for $(x, \lambda, w) \in \mathbb{R}^{n} \times \mathbb{R}_{++}^{m} \times \mathbb{R}_{++}^{m}$;

(F7) $J \Psi(x, \lambda)$ is nonsingular for $(x, \lambda) \in \mathbb{R}^{n} \times \mathbb{R}_{++}^{m}$ with $h(x)<0$.

To the best of our knowledge, the literature of QVIs and GNEPs has only investigated conditions guaranteeing facts (F1) and (F6) so far; see [19,21]. Constrained stationary points have been considered in the case of variational inequalities in [34], but not for QVIs yet.

The results we will prove are of great practical interest. On the one hand, for the first time, we give conditions guaranteeing that finding a stationary point of the merit function $\frac{1}{2}\|H(x, \lambda, w)\|^{2}$ subject to $\lambda \geq 0$ and $w \geq 0$, or the merit function $\frac{1}{2}\|\Psi(x, \lambda)\|^{2}$ subject to $\lambda \geq 0$, we are actually computing solutions of the QVI. On the other hand, an important hint given by this study is that if we want to solve the QVI by adopting a projected gradient-type method designed to minimize the merit functions described above, then reformulation (2) is the best option. While if we want to resort to a second order interior point algorithm, reformulation (3) seems to be preferable.

Before stating the assumptions we use in our analysis, let us introduce some notation. For an index set $J \subseteq\{1, \ldots, m\}$, we write $\nabla_{y} g_{\bullet} J(x, x)$ for the matrix with all columns of $\nabla_{y} g(x, x)$ that belong to $J$, and by $J h_{J \bullet}(x)$ we denote the matrix with all rows of $J h(x)$ with indices in $J$.

These are our main assumptions: 
(A1) For all $(x, \lambda) \in \mathbb{R}^{n} \times \mathbb{R}^{m}$, the matrix $J_{x} L(x, \lambda)$ is nonsingular and the matrix $M(x, \lambda)$ is a $P_{0}$ matrix.

(A1+) For all $(x, \lambda) \in \mathbb{R}^{n} \times \mathbb{R}_{+}^{m}$, the matrix $J_{x} L(x, \lambda)$ is nonsingular and the matrix $M(x, \lambda)$ is a $P_{0}$ matrix.

$(\mathrm{A} 1++)$ For all $(x, \lambda) \in \mathbb{R}^{n} \times \mathbb{R}_{++}^{m}$, the matrix $J_{x} L(x, \lambda)$ is nonsingular and the matrix $M(x, \lambda)$ is a $P_{0}$ matrix.

(A2) For all $(x, \lambda) \in \mathbb{R}^{n} \times \mathbb{R}_{+}^{m}$, the matrix $J_{x} L(x, \lambda)$ is nonsingular and the matrix $M(x, \lambda)$ is a semimonotone matrix.

(A3) For all $(x, \lambda) \in \mathbb{R}^{n} \times \mathbb{R}_{+}^{m}$, the matrix $J_{x} L(x, \lambda)$ is nonsingular and $M(x, \lambda)$ is a positive semidefinite $S$ matrix.

(A4) All the constraints are of the form $g(y, x)=A y+c(x)$, with a matrix $A \in \mathbb{R}^{m \times n}$ and a function $c: \mathbb{R}^{n} \rightarrow \mathbb{R}^{m}$.

(A5) Let (A4) hold and let an $x \in \mathbb{R}^{n}$ be given. Set

$$
d_{J}:=\operatorname{det}\left(\begin{array}{cc}
J_{x} F(x) & \nabla_{y} g_{\bullet} J \\
-J h_{J \bullet}(x) & 0
\end{array}\right)
$$

We assume for some index set $J \subseteq\{1, \ldots, m\}$ that $d_{J} \neq 0$ and either $d_{J} \geq 0$ for all $J \subseteq\{1, \ldots, m\}$, or $d_{J} \leq 0$ for all $J \subseteq\{1, \ldots, m\}$.

Note that the first three assumptions only differ in the range of $\lambda$, and (A1) implies $(\mathrm{A} 1+)$, which again implies $(\mathrm{A} 1++)$. Since every positive semidefinite matrix is also a $P_{0}$ matrix, (A3) implies (A1+). Since every $P_{0}$ matrix is a semimonotone matrix, (A1+) implies (A2). In (A4), we refer to a class of QVIs for which the constraints are separable in $x$ and $y$ and where the $y$-part is linear. This is, in particular, satisfied for linear constraints. In [21], constraints of QVIs for which (A4) holds are termed linear with variable right-hand side. In this case, we obtain

$$
J h(x)=A+J c(x), \quad \nabla_{y} g(x, x)=\nabla_{y} g=A^{\top} \quad \text { and } \quad J_{x} L(x, \lambda)=J_{x} F(x),
$$

and we see that both $J_{x} L(x, \lambda)$ and $M(x, \lambda)$ become independent of the multipliers $\lambda$.

In Figs. 1 and 2, we summarize the relations we show in the next two sections.

\section{Stationarity Results for QVIs}

In this section, we want to see how (A1)-(A3) imply (F1)-(F5). For (unconstrained) stationarity results, it is clear that nonsingularity of the Jacobian, or of all the elements of the generalized Jacobian, implies that these points must be zeros of the function. Conditions ensuring this will be shown in the next section. Here, we present conditions not requiring this nonsingularity. First, we generalization the GNEP result [19, Theorem 3.1] to QVIs.

Proposition 3.1 If (A1) holds, every stationarypoint of the merit function $\frac{1}{2}\|\Psi(x, \lambda)\|^{2}$ is a zero of $\Psi$, i.e., (F1) is true. 


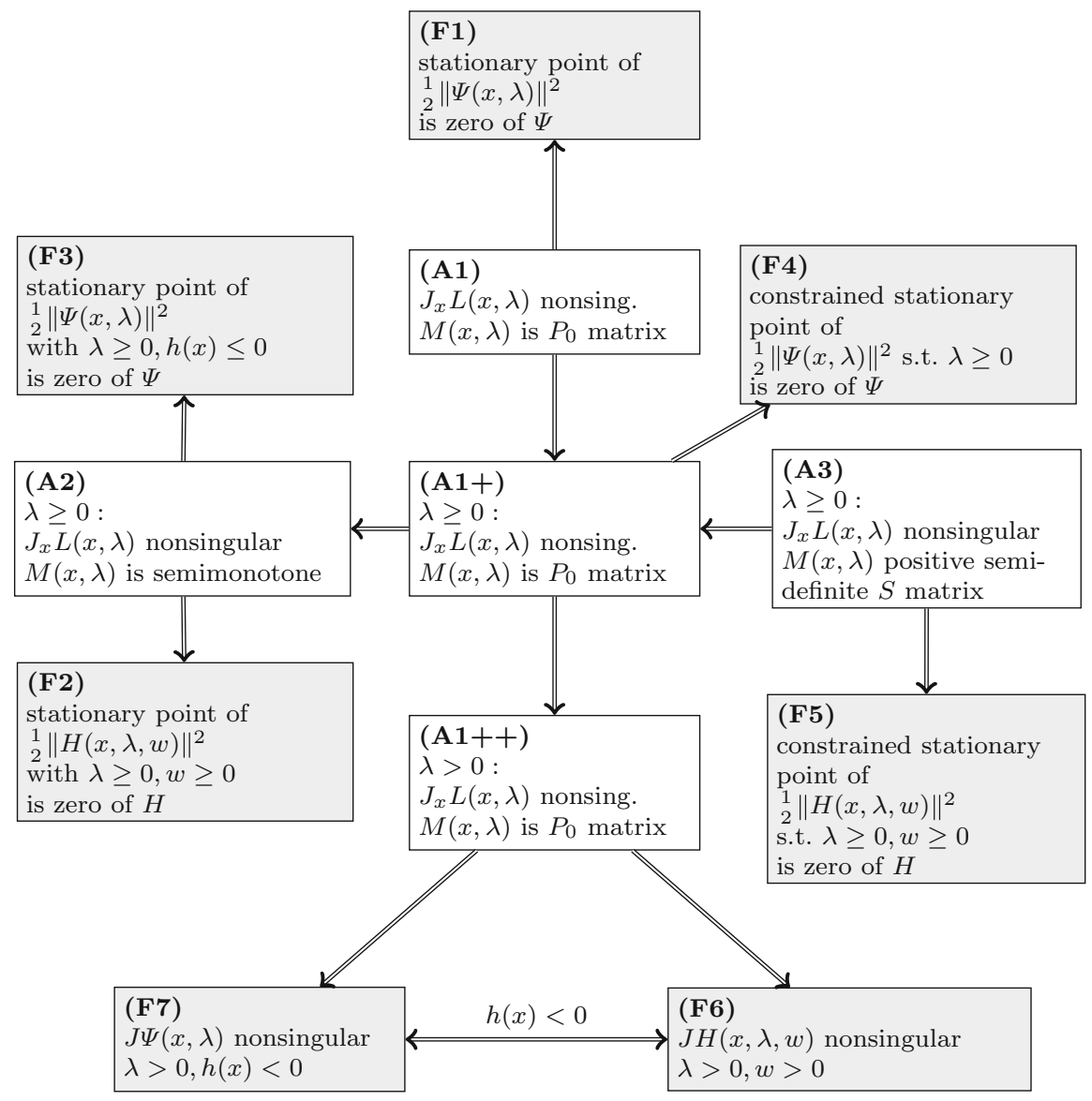

Fig. 1 Summary of relations between assumptions (A1)-(A3) and facts (F1)-(F7)

Proof Let $(x, \lambda) \in \mathbb{R}^{n} \times \mathbb{R}^{m}$ be a stationary point, meaning

$$
0=\psi^{\top} \Psi(x, \lambda)=\left(\begin{array}{cc}
\nabla_{x} L(x, \lambda) & -\nabla h(x) D_{h} \\
J_{y} g(x, x) & D_{\lambda}
\end{array}\right)\left(\begin{array}{c}
L(x, \lambda) \\
\varphi(\lambda,-h(x))
\end{array}\right),
$$

where $\psi \in \partial \Psi(x, \lambda)$. Using nonsingularity of $J_{x} L(x, \lambda)$ we get

$$
L(x, \lambda)=\nabla_{x} L(x, \lambda)^{-1} \nabla h(x) D_{h} \varphi(\lambda,-h(x)) .
$$

With the definition of $M(x, \lambda)$ from (4), we obtain

$$
\left(M(x, \lambda)^{\top} D_{h}+D_{\lambda}\right)(\varphi(\lambda,-h(x)))=0 .
$$

Both $D_{h}$ and $D_{\lambda}$ can be assumed to be negative diagonal matrices, because they are nonpositive and $D_{h}^{i}$ or $D_{\lambda}^{i}$ can be zero only if $\varphi\left(\lambda_{i},-h_{i}(x)\right)=0$. With $M(x, \lambda)$ also its 


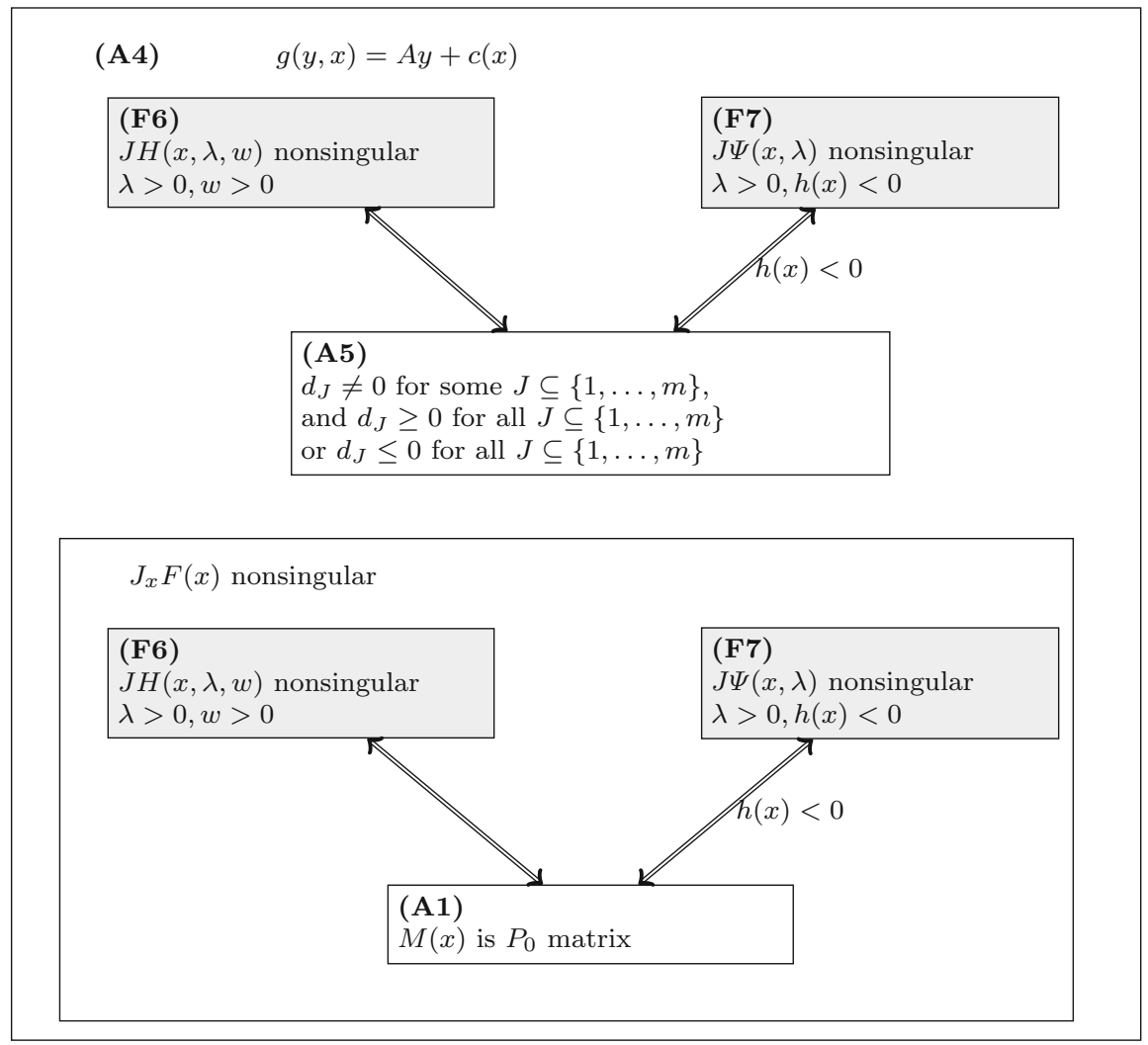

Fig. 2 Equivalence relations between (F6) and (F7) to (A1) and (A5) under (A4)

transposed is a $P_{0}$ matrix by (A1). From [35], we get that $M(x, \lambda)^{\top}$ being a $P_{0}$ matrix and $D_{h}, D_{\lambda}$ being negative definite implies $M(x, \lambda)^{\top} D_{h}+D_{\lambda}$ being nonsingular. This yields $\varphi(\lambda,-h(x))=0$, and then, $L(x, \lambda)=0$, which completes the proof.

The following results are useful to guarantee that unconstrained stationary points, whose dual variables are nonnegative, are solutions of the QVI.

Proposition 3.2 If (A2) holds, every stationary point of $\frac{1}{2}\|H(x, \lambda, w)\|^{2}$, for which $\lambda \geq 0$ and $w \geq 0$ holds, is a zero of $H$, i.e., (F2) is true.

Proof Let $(x, \lambda, w) \in \mathbb{R}^{n} \times \mathbb{R}_{+}^{m} \times \mathbb{R}_{+}^{m}$ be a stationary point, meaning $0=J H(x, \lambda, w)^{\top} H(x, \lambda, w)=\left(\begin{array}{ccc}\nabla_{x} L(x, \lambda) & \nabla h(x) & 0 \\ J_{y} g(x, x) & 0 & \operatorname{diag}(w) \\ 0 & I & \operatorname{diag}(\lambda)\end{array}\right)\left(\begin{array}{c}L(x, \lambda) \\ h(x)+w \\ \lambda \circ w\end{array}\right)$

This yields

$$
h(x)+w=-\operatorname{diag}(\lambda)(\lambda \circ w),
$$


and, using nonsingularity of $J_{x} L(x, \lambda)$ from (A2),

$$
L(x, \lambda)=-\nabla_{x} L(x, \lambda)^{-1} \nabla h(x)(h(x)+w) .
$$

Inserting this in

$$
J_{y} g(x, x) L(x, \lambda)+\operatorname{diag}(w)(\lambda \circ w)=0,
$$

we obtain

$$
\left(M(x, \lambda)^{\top} \operatorname{diag}(\lambda)+\operatorname{diag}(w)\right)(\lambda \circ w)=0 .
$$

Let us assume for contradiction that $\lambda \circ w \neq 0$. With $\operatorname{diag}(\lambda) \in \mathbb{R}_{+}^{m}$ and $M(x, \lambda)$ being a semimonotone matrix, also $M(x, \lambda)^{\top}$ and $M(x, \lambda)^{\top} \operatorname{diag}(\lambda)$ are semimonotone matrices. By definition, an index $j \in\{1, \ldots, m\}$ exists such that

$$
\lambda_{j} w_{j}>0, \quad\left(M(x, \lambda)^{\top} \operatorname{diag}(\lambda)(\lambda \circ w)\right)_{j} \geq 0 .
$$

This implies the contradiction

$$
\begin{aligned}
0 & =\left(\left(M(x, \lambda)^{\top} \operatorname{diag}(\lambda)+\operatorname{diag}(w)\right)(\lambda \circ w)\right)_{j} \\
& =\left(M(x, \lambda)^{\top} \operatorname{diag}(\lambda)(\lambda \circ w)\right)_{j}+\lambda_{j} w_{j}^{2}>0 .
\end{aligned}
$$

Thus, we must have $\lambda \circ w=0$, and the proof holds by observing that we also get $h(x)+w=0$ and $L(x, \lambda)=0$.

For illustration, we present the following example where nonoptimal stationary points, with nonnegative $\lambda$ and $w$, are present.

Example 3.1 Define $F: \mathbb{R}^{2} \rightarrow \mathbb{R}^{2}$ and $g: \mathbb{R}^{2} \times \mathbb{R}^{2} \rightarrow \mathbb{R}^{2}$ by

$$
F(x)=\left(\begin{array}{l}
2 x_{2}-\frac{9}{2} \\
2 x_{1}-\frac{9}{2}
\end{array}\right) \quad \text { and } \quad g(y, x)=\left(\begin{array}{l}
-3 x_{1}+2 y_{2}-1 \\
-3 x_{2}+2 y_{1}-1
\end{array}\right) .
$$

Here, every stationary point of $\frac{1}{2}\|H(x, \lambda, w)\|^{2}$ satisfies

$$
\begin{aligned}
0 & =J H(x, \lambda, w)^{\top} H(x, \lambda, w) \\
& =\left(\begin{array}{cccccc}
0 & 2 & -3 & 2 & 0 & 0 \\
2 & 0 & 2 & -3 & 0 & 0 \\
0 & 2 & 0 & 0 & w_{1} & 0 \\
2 & 0 & 0 & 0 & 0 & w_{2} \\
0 & 0 & 1 & 0 & \lambda_{1} & 0 \\
0 & 0 & 0 & 1 & 0 & \lambda_{2}
\end{array}\right)\left(\begin{array}{c}
2 x_{2}-\frac{9}{2}+2 \lambda_{2} \\
2 x_{1}-\frac{9}{2}+2 \lambda_{1} \\
-3 x_{1}+2 x_{2}-1+w_{1} \\
2 x_{1}-3 x_{2}-1+w_{2} \\
\lambda_{1} w_{1} \\
\lambda_{2} w_{2}
\end{array}\right) .
\end{aligned}
$$


Now one can check that the point $x=(1,1), \lambda=(1,1)$ and $w=(1,1)$ is a stationary point, but it is certainly not a zero of $H$. In this example, we have nonsingularity of the matrix $J_{x} L(x, \lambda)=\left(\begin{array}{ll}0 & 2 \\ 2 & 0\end{array}\right)$, but the matrix

$$
M(x, \lambda)=\left(\begin{array}{cc}
-3 & 2 \\
2 & -3
\end{array}\right)\left(\begin{array}{ll}
0 & \frac{1}{2} \\
\frac{1}{2} & 0
\end{array}\right)\left(\begin{array}{ll}
0 & 2 \\
2 & 0
\end{array}\right)=\left(\begin{array}{cc}
-3 & 2 \\
2 & -3
\end{array}\right)
$$

is not semimonotone.

Assumption (A2) can also be used for the nonsmooth reformulation to get (F3), as we show next.

Proposition 3.3 Let (A2) hold. Then, every stationary point of $\frac{1}{2}\|\Psi(x, \lambda)\|^{2}$ satisfying $\lambda \geq 0$ and $h(x) \leq 0$ is a zero of $\Psi$, i.e., $(\mathrm{F} 3)$ is true.

Proof As in Proposition 3.1, we obtain (5) that in turn can be written as

$$
\left(M(x, \lambda)^{\top}\left(-D_{h}\right)+\left(-D_{\lambda}\right)\right)(-\varphi(\lambda,-h(x)))=0 .
$$

By (A2), $-D_{h}$ being a nonnegative diagonal matrix implies that the matrix $M(x, \lambda)^{\top}\left(-D_{h}\right)$ is semimonotone. Let us assume by contradiction that $-\varphi(\lambda,-h(x)) \neq 0$. Observing that $-\varphi(\lambda,-h(x)) \geq 0$ for all $\lambda \geq 0$ and $h(x) \leq 0$, the definition of semimonotonicity yields the existence of an index $j \in\{1, \ldots, m\}$ such that

$$
-\varphi\left(\lambda_{j},-h_{j}(x)\right)>0, \quad\left(M(x, \lambda)^{\top}\left(-D_{h}\right)(-\varphi(\lambda,-h(x)))\right)_{j} \geq 0
$$

This implies the contradiction

$$
\begin{aligned}
0= & \left(\left(M(x, \lambda)^{\top}\left(-D_{h}\right)+\left(-D_{\lambda}\right)\right)(-\varphi(\lambda,-h(x)))\right)_{j} \\
= & \left(M(x, \lambda)^{\top}\left(-D_{h}\right)(-\varphi(\lambda,-h(x)))\right)_{j} \\
& +\left(\frac{\lambda_{j}}{\sqrt{\lambda_{j}^{2}+h_{j}(x)^{2}}}-1\right) \varphi\left(\lambda_{j},-h_{j}(x)\right)>0,
\end{aligned}
$$

where the last inequality follows since $\varphi\left(\lambda_{j},-h_{j}(x)\right)<0$ implies $\lambda_{j}>0$ and $h_{j}(x)<0$. Thus, we must have $\varphi(\lambda,-h(x))=0$, and then, nonsingularity of $J_{x} L(x, \lambda)$ implies $L(x, \lambda)=0$ and $\Psi(x, \lambda)=0$.

In the following part, we consider constrained stationary points. For the merit function $\frac{1}{2}\|\Psi(x, \lambda)\|^{2}$, we obtain (F4) under (A1+).

Theorem 3.1 Let (A1+) hold. Then, every constrained stationary point of $\frac{1}{2}\|\Psi(x, \lambda)\|^{2}$ subject to $\lambda \geq 0$ is a zero of $\Psi$, i.e., (F4) is true. 
Proof Let $(x, \lambda) \in \mathbb{R}^{n} \times \mathbb{R}_{+}^{m}$ be a constrained stationary point, meaning

$$
\begin{aligned}
& \nabla_{x} L(x, \lambda) L(x, \lambda)-\nabla h(x) D_{h} \varphi(\lambda,-h(x))=0, \\
& \lambda_{i}>0 \Rightarrow \nabla_{y} g_{i}(x, x)^{\top} L(x, \lambda)+D_{\lambda}^{i} \varphi\left(\lambda_{i},-h_{i}(x)\right)=0, \\
& \lambda_{i}=0 \Rightarrow \nabla_{y} g_{i}(x, x)^{\top} L(x, \lambda)+D_{\lambda}^{i} \varphi\left(\lambda_{i},-h_{i}(x)\right) \geq 0 .
\end{aligned}
$$

The nonsingularity of $J_{x} L(x, \lambda)$ from $(\mathrm{A} 1+)$ implies

$$
L(x, \lambda)=\nabla_{x} L(x, \lambda)^{-1} \nabla h(x) D_{h} \varphi(\lambda,-h(x)) .
$$

Hence, if we show that $\varphi(\lambda,-h(x))=0$, we also get $L(x, \lambda)=0$. Let us recall that whenever $\varphi\left(\lambda_{i},-h_{i}(x)\right) \neq 0$, the diagonal elements $D_{h}^{i}$ and $D_{\lambda}^{i}$ are negative. Now, we replace $L(x, \lambda)$ in the constrained stationarity conditions by the above formula, use the definition of $M(x, \lambda)$ from (4) and consider five possible cases:

(a) For $\lambda_{i}=0, h_{i}(x) \leq 0$, we have $\varphi\left(\lambda_{i},-h_{i}(x)\right)=0$ and $D_{h}^{i} \varphi\left(\lambda_{i},-h_{i}(x)\right)=0$.

(b) For $\lambda_{i}>0, h_{i}(x)=0$, we have $\varphi\left(\lambda_{i},-h_{i}(x)\right)=0$ and $D_{h}^{i} \varphi\left(\lambda_{i},-h_{i}(x)\right)=0$.

(c) For $\lambda_{i}=0, h_{i}(x)>0$, we have $\varphi\left(\lambda_{i},-h_{i}(x)\right)>0$ and the stationarity conditions imply

$$
\left(M_{\bullet i}(x, \lambda)\right)^{\top} D_{h} \varphi(\lambda,-h(x)) \geq-D_{\lambda}^{i} \varphi\left(\lambda_{i},-h_{i}(x)\right)>0 .
$$

(d) For $\lambda_{i}>0, h_{i}(x)<0$ we have $\varphi\left(\lambda_{i},-h_{i}(x)\right)<0$ and the stationarity conditions imply

$$
\left(M_{\bullet i}(x, \lambda)\right)^{\top} D_{h} \varphi(\lambda,-h(x))=-D_{\lambda}^{i} \varphi\left(\lambda_{i},-h_{i}(x)\right)<0 .
$$

(e) For $\lambda_{i}>0, h_{i}(x)>0$, we have $\varphi\left(\lambda_{i},-h_{i}(x)\right)>0$ and the stationarity conditions imply

$$
\left(M_{\bullet i}(x, \lambda)\right)^{\top} D_{h} \varphi(\lambda,-h(x))=-D_{\lambda}^{i} \varphi\left(\lambda_{i},-h_{i}(x)\right)>0 .
$$

Together, we have for all $i \in\{1, \ldots, m\}$ either $D_{h}^{i} \varphi\left(\lambda_{i},-h_{i}(x)\right)=0$ or

$$
\left(\varphi\left(\lambda_{i},-h_{i}(x)\right) D_{h}^{i}\right)\left(M_{\bullet i}(x, \lambda)\right)^{\top} D_{h} \varphi(\lambda,-h(x))<0 .
$$

With $M(x, \lambda)$ also its transposed matrix is a $P_{0}$ matrix. If $D_{h} \varphi(\lambda,-h(x)) \neq 0$, the definition of a $P_{0}$ matrix implies that for at least one index $j \in\{1, \ldots, m\}$ we have $D_{h}^{j} \varphi\left(\lambda_{j},-h_{j}(x)\right) \neq 0$ and

$$
\left(\varphi\left(\lambda_{j},-h_{j}(x)\right) D_{h}^{j}\right)\left(M_{\bullet j}(x, \lambda)\right)^{\top} D_{h} \varphi(\lambda,-h(x)) \geq 0 .
$$

This condition excludes cases (c) to (e), and we must have $\varphi(\lambda,-h(x))=0$. This completes the proof. 
In order to obtain (F5), we use the stronger assumption (A3).

Theorem 3.2 If (A3) holds, every constrained stationary point of the merit function $\frac{1}{2}\|H(x, \lambda, w)\|^{2}$ subject to $\lambda \geq 0$ and $w \geq 0$ is a zero of $H$, i.e., (F5) is true.

Proof Let $(x, \lambda, w) \in \mathbb{R}^{n} \times \mathbb{R}_{+}^{m} \times \mathbb{R}_{+}^{m}$ be a constrained stationary point, meaning:

$$
\begin{aligned}
& \nabla_{x} L(x, \lambda) L(x, \lambda)+\nabla h(x)(h(x)+w)=0, \\
& \lambda_{i}>0 \Rightarrow \nabla_{y} g_{i}(x, x)^{\top} L(x, \lambda)+w_{i}^{2} \lambda_{i}=0 \\
& \lambda_{i}=0 \Rightarrow \nabla_{y} g_{i}(x, x)^{\top} L(x, \lambda)+w_{i}^{2} \lambda_{i} \geq 0 \\
& w_{i}>0 \Rightarrow h_{i}(x)+w_{i}+w_{i} \lambda_{i}^{2}=0 \\
& w_{i}=0 \Rightarrow h_{i}(x)+w_{i}+w_{i} \lambda_{i}^{2} \geq 0 .
\end{aligned}
$$

Nonsingularity of $J_{x} L(x, \lambda)$ implies $L(x, \lambda)=-\nabla_{x} L(x, \lambda)^{-1} \nabla h(x)(h(x)+w)$. We must consider four possible cases:

(a) For $\lambda_{i}>0, w_{i}>0$, we get

$$
\left(M_{\bullet i}(x, \lambda)\right)^{\top}(h(x)+w)=w_{i}^{2} \lambda_{i}>0, \quad h_{i}(x)+w_{i}=-w_{i} \lambda_{i}^{2}<0 .
$$

(b) For $\lambda_{i}>0, w_{i}=0$, we get

$$
\left(M_{\bullet i}(x, \lambda)\right)^{\top}(h(x)+w)=w_{i}^{2} \lambda_{i}=0, \quad h_{i}(x)+w_{i} \geq-w_{i} \lambda_{i}^{2}=0 .
$$

(c) For $\lambda_{i}=0, w_{i}>0$, we get

$$
\left(M_{\bullet i}(x, \lambda)\right)^{\top}(h(x)+w) \leq w_{i}^{2} \lambda_{i}=0, \quad h_{i}(x)+w_{i}=-w_{i} \lambda_{i}^{2}=0 .
$$

(d) For $\lambda_{i}=0, w_{i}=0$, we get

$$
\left(M_{\bullet i}(x, \lambda)\right)^{\top}(h(x)+w) \leq w_{i}^{2} \lambda_{i}=0, \quad h_{i}(x)+w_{i} \geq-w_{i} \lambda_{i}^{2}=0 .
$$

Thus, in all the cases we obtain

$$
\left(h_{i}(x)+w_{i}\right)\left(M_{\bullet i}(x, \lambda)\right)^{\top}(h(x)+w) \leq 0 .
$$

Since $M(x, \lambda)$, and hence also its transposed matrix, is positive semidefinite, we get

$$
\begin{aligned}
0 & \geq \sum_{i=1}^{m}\left(h_{i}(x)+w_{i}\right)\left(M_{\bullet i}(x, \lambda)\right)^{\top}(h(x)+w) \\
& =(h(x)+w)(M(x, \lambda))^{\top}(h(x)+w) \geq 0 .
\end{aligned}
$$

This implies that for all $i=1, \ldots, m$ we must have

$$
\left(h_{i}(x)+w_{i}\right)\left(M_{\bullet i}(x, \lambda)\right)^{\top}(h(x)+w)=0 .
$$


Thus, case (a) cannot hold, and we have $\lambda \circ w=0$. By (A3), $M(x, \lambda)$ is an $S$ matrix, and hence, the system $M(x, \lambda) z>0, z>0$ is solvable. The alternatives theorem of Ville, see [36], gives that the system

$$
M(x, \lambda)^{\top} v \leq 0, \quad v \geq 0, \quad v \neq 0
$$

is not solvable. From the cases (b) to (d), we have that

$$
M(x, \lambda)^{\top}(h(x)+w) \leq 0, \quad h(x)+w \geq 0 .
$$

Hence, we must have $h(x)+w=0$. This, in turn implies $L(x, \lambda)=0$, and therefore, we have $H(x, \lambda, w)=0$.

Inspecting the proof starting from equation (7), one could also finalize it by assuming that $M(x, \lambda)$ is a $P$ matrix. Unfortunately, the $P$ property of $M(x, \lambda)$ for all $\lambda \in \mathbb{R}_{+}^{m}$ is a very strong condition, since it implies nonsingularity of the matrix $M(x, \lambda)$. This, in turn, can only hold if $\nabla h(x)$ and $J_{y} g(x, x)$ are full rank matrices and hence, in particular, requires that we have linear independent constraints.

Let us give an example, where we can apply Theorem 3.2.

Example 3.2 We consider the QVI defined through

$$
F(x):=\left(\begin{array}{l}
2 x_{1} \\
2 x_{2} \\
2 x_{3}
\end{array}\right), \quad g(y, x):=\left(\begin{array}{c}
2 y_{1}+x_{2}+2 x_{3}+1 \\
x_{1}+2 y_{2}+x_{3}+2 \\
2 x_{1}+x_{2}+2 y_{3}+3
\end{array}\right) .
$$

Here, the matrix $J_{x} L(x, \lambda)=J_{x} F$ is nonsingular. Further, we have

$$
M=\left(\begin{array}{lll}
2 & 1 & 2 \\
1 & 2 & 1 \\
2 & 1 & 2
\end{array}\right)\left(\begin{array}{ccc}
1 / 2 & 0 & 0 \\
0 & 1 / 2 & 0 \\
0 & 0 & 1 / 2
\end{array}\right)\left(\begin{array}{lll}
2 & 0 & 0 \\
0 & 2 & 0 \\
0 & 0 & 2
\end{array}\right)=\left(\begin{array}{lll}
2 & 1 & 2 \\
1 & 2 & 1 \\
2 & 1 & 2
\end{array}\right)
$$

which is symmetric positive semidefinite. Since all matrix entries of $M$ are positive, the system $M z>0, z>0$ can be solved with a vector having all elements equal to 1 , and $M$ is an $S$ matrix. Thus, Theorem 3.2 guarantees that (F5) holds.

With the next example, we see that, in contrast to (F4), (A1+) or positive semidefiniteness of $M(x, \lambda)$ alone is not sufficient to get (F5).

Example 3.3 Let us consider the QVI defined through

$$
F(x):=\left(\begin{array}{l}
x_{1} \\
x_{2}
\end{array}\right), \quad g(y, x):=\left(\begin{array}{c}
y_{1}+x_{2}+2 \\
-2 x_{1}-2 y_{2}+1
\end{array}\right) .
$$

This QVI does not even have a feasible point, but a constrained stationary point $\bar{x}=(0,0), \bar{\lambda}=(0,0), \bar{w}=(0,0)$, since

$$
L(\bar{x}, \bar{\lambda})=\left(\begin{array}{l}
0 \\
0
\end{array}\right), \quad h(\bar{x})=\left(\begin{array}{l}
2 \\
1
\end{array}\right), \quad \text { and } \nabla h(\bar{x})=\left(\begin{array}{l}
1-2 \\
1-2
\end{array}\right),
$$


imply the constrained stationarity conditions

$$
\begin{aligned}
& \\
& \nabla_{x} L(\bar{x}, \bar{\lambda}) L(\bar{x}, \bar{\lambda})+\nabla h(\bar{x})(h(\bar{x})+\bar{w})=0, \\
& \lambda_{i}=0 \Rightarrow \nabla_{y} g_{i}(\bar{x}, \bar{x})^{\top} L(\bar{x}, \bar{\lambda})+\bar{w}_{i}^{2} \bar{\lambda}_{i} \geq 0 \\
& \bar{w}_{i}=0 \Rightarrow h_{i}(\bar{x})+\bar{w}_{i}+\bar{w}_{i} \bar{\lambda}_{i}^{2} \geq 0 .
\end{aligned}
$$

However, this point is not feasible, hence not a solution of $H(x, \lambda, w)=0$. Furthermore, in this example we have

$$
M=\left(\begin{array}{cc}
1 & 1 \\
-2 & -2
\end{array}\right)\left(\begin{array}{ll}
1 & 0 \\
0 & 1
\end{array}\right)\left(\begin{array}{cc}
1 & 0 \\
0 & -2
\end{array}\right)=\left(\begin{array}{cc}
1 & -2 \\
-2 & 4
\end{array}\right),
$$

which is a $P_{0}$ matrix and positive semidefinite. This shows that the $P_{0}$ property of $M(x, \lambda)$, or its positive semidefiniteness, is not sufficient for a constrained stationary point of $H$ to be a solution of $H(x, \lambda, w)=0$.

We can also give an example that has feasible points and where $M$ is positive semidefinite, but nonoptimal constrained stationary points exist.

Example 3.4 Consider the QVI defined through

$$
F(x):=\left(\begin{array}{c}
x_{1} \\
x_{2}+3
\end{array}\right), \quad g(y, x):=\left(\begin{array}{c}
y_{1}-2 x_{2}+2 \\
-2 y_{1}+x_{2}+1
\end{array}\right) .
$$

This QVI has feasible points, for example $(2,2)$. Here, the point $\bar{x}=(0,0)$, $\bar{\lambda}=(0,0), \bar{w}=(0,0)$ is a constrained stationary point, since

$$
\begin{aligned}
& h(x)+w=\left(\begin{array}{l}
2 \\
1
\end{array}\right), \quad L=\left(\begin{array}{l}
0 \\
3
\end{array}\right), \quad \nabla_{y} g^{\top} L=\left(\begin{array}{l}
0 \\
0
\end{array}\right) \\
& \nabla_{x} L(\bar{x}, \bar{\lambda}) L(\bar{x}, \bar{\lambda})+\nabla h(\bar{x})(h(\bar{x})+w)=\left(\begin{array}{l}
0 \\
3
\end{array}\right)+\left(\begin{array}{cc}
1 & -2 \\
-2 & 1
\end{array}\right)\left(\begin{array}{l}
2 \\
1
\end{array}\right)=\left(\begin{array}{l}
0 \\
0
\end{array}\right) .
\end{aligned}
$$

Obviously the point is not feasible and hence not a zero of $H$. We have in this example

$$
M=\left(\begin{array}{cc}
1 & -2 \\
-2 & 1
\end{array}\right)\left(\begin{array}{cc}
1 & -2 \\
0 & 0
\end{array}\right)=\left(\begin{array}{cc}
1 & -2 \\
-2 & 4
\end{array}\right),
$$

which is a symmetric positive semidefinite matrix. Hence, this condition is not sufficient for (F5). Moreover, we can see that (A3) is violated, since the system $M z>0, z>0$ is not solvable.

\section{Nonsingularity Results for QVIs}

In this section, we deal with the nonsingularity facts (F6) and (F7). Let us recall that for $\lambda \in \mathbb{R}_{++}^{m}$ the set $\partial \Psi(x, \lambda)=\{J \Psi(x, \lambda)\}$ is single valued. First, we show that the two facts (F6) and (F7) are equivalent for all $x$ with $h(x)<0$. 
Proposition 4.1 Let an arbitrary $x \in \mathbb{R}^{n}$ with $h(x)<0$ be given. Then, $J \Psi(x, \lambda)$ is nonsingular for all $\lambda \in \mathbb{R}_{++}^{m}$ if and only if $J H(x, \lambda, w)$ is nonsingular for all $(\lambda, w) \in \mathbb{R}_{++}^{m} \times \mathbb{R}_{++}^{m}$. This means $(\mathrm{F} 7)$ is equivalent to (F6) for all $x \in \mathbb{R}^{n}$ with $h(x)<0$.

Proof For $h(x)<0$ and $\lambda \in \mathbb{R}_{++}^{m}$, the matrices $D_{h}$ and $D_{\lambda}$ are negative definite, with $D_{h}^{i}=\frac{-h_{i}(x)}{\sqrt{h_{i}(x)^{2}+\lambda_{i}^{2}}}-1<0$ and $D_{\lambda}^{i}=\frac{\lambda_{i}}{\sqrt{h_{i}(x)^{2}+\lambda_{i}^{2}}}-1<0$. Thus, the matrix $J \Psi(x, \lambda)=$ $\left(\begin{array}{cc}J_{x} L(x, \lambda) & \nabla_{y} g(x, x) \\ -D_{h} J h(x) & D_{\lambda}\end{array}\right)$ is nonsingular, if and only if $\left(\begin{array}{cc}J_{x} L(x, \lambda) & \nabla_{y} g(x, x) \\ J h(x) & -D_{h}^{-1} D_{\lambda}\end{array}\right)$ is nonsingular. For any fixed $x \in \mathbb{R}^{n}$ with $h_{i}(x)<0$ and $\lambda_{i}>0$, the function

$$
\lambda_{i} \mapsto\left(D_{h}^{i}\right)^{-1}\left(D_{\lambda}^{i}\right)=\frac{\lambda_{i}-\sqrt{h_{i}(x)^{2}+\lambda_{i}^{2}}}{-h_{i}(x)-\sqrt{h_{i}(x)^{2}+\lambda_{i}^{2}}} .
$$

is continuous with $\lim _{\lambda_{i} \downarrow 0}\left(D_{h}^{i}\right)^{-1}\left(D_{\lambda}^{i}\right)=+\infty$ and $\lim _{\lambda_{i} \rightarrow \infty}\left(D_{h}^{i}\right)^{-1}\left(D_{\lambda}^{i}\right)=0$. Thus, nonsingularity of $J \Psi(x, \lambda)$ for all $\lambda \in \mathbb{R}_{++}^{m}$ is equivalent to nonsingularity of the matrices

$$
\left(\begin{array}{cc}
J_{x} L(x, \lambda) & \nabla_{y} g(x, x) \\
J h(x) & -D
\end{array}\right)
$$

for all positive definite diagonal matrices $D \in \mathbb{R}_{++}^{m \times m}$.

Now, since for some $(\lambda, w) \in \mathbb{R}_{++}^{m} \times \mathbb{R}_{++}^{m}$ we can write any positive definite diagonal matrix in the form $D=\operatorname{diag}(\lambda)^{-1} \operatorname{diag}(w)$, nonsingularity of (9) for all positive definite diagonal matrices $D \in \mathbb{R}_{++}^{m \times m}$ is equivalent to nonsingularity of

$$
\left(\begin{array}{cc}
J_{x} L(x, \lambda) & \nabla_{y} g(x, x) \\
J h(x) & -\operatorname{diag}(\lambda)^{-1} \operatorname{diag}(w)
\end{array}\right)
$$

for all $(\lambda, w) \in \mathbb{R}_{++}^{m} \times \mathbb{R}_{++}^{m}$. This, in turn, is equivalent to

$$
J H(x, \lambda, w)=\left(\begin{array}{ccc}
J_{x} F(x) & \nabla_{y} g & 0 \\
J h(x) & 0 & I \\
0 & \operatorname{diag}(w) & \operatorname{diag}(\lambda)
\end{array}\right)
$$

being nonsingular for all $(\lambda, w) \in \mathbb{R}_{++}^{m} \times \mathbb{R}_{++}^{m}$, and the proof is complete.

The following general necessary and sufficient condition for the nonsingularity of $J H$ was proved in [21, Theorem 3].

Proposition 4.2 Let $(x, \lambda, w) \in \mathbb{R}^{n} \times \mathbb{R}_{++}^{m} \times \mathbb{R}_{++}^{m}$ be given. Then, the matrix

$$
J_{x} L(x, \lambda)+\nabla_{y} g(x, x) \operatorname{diag}\left(w^{-1} \circ \lambda\right) J h(x)
$$

is nonsingular, if and only if $J H(x, \lambda, w)$ is nonsingular. 
In the same spirit, we obtain a similar result for $J \Psi$.

Proposition 4.3 Let $(x, \lambda) \in \mathbb{R}^{n} \times \mathbb{R}_{++}^{m}$ with $h(x) \neq 0$ be given. Then, the matrix

$$
J_{x} L(x, \lambda)+\nabla_{y} g(x, x) D_{\lambda}^{-1} D_{h} J h(x)
$$

is nonsingular, if and only if $J \Psi(x, \lambda)$ is nonsingular.

Proof Since $h(x) \neq 0$ and $\lambda \in \mathbb{R}_{++}^{m}$, we have a negative definite diagonal matrix $D_{\lambda}$. For a vector $v=\left(v^{1}, v^{2}\right) \in \mathbb{R}^{n+m}$, we get that

$$
0=J \Psi(x, \lambda) v=\left(\begin{array}{cc}
J_{x} L(x, \lambda) & \nabla_{y} g(x, x) \\
-D_{h} J h(x) & D_{\lambda}
\end{array}\right)\left(\begin{array}{l}
v^{1} \\
v^{2}
\end{array}\right)
$$

is equivalent to

$$
\begin{aligned}
v^{2} & =D_{\lambda}^{-1} D_{h} J h(x) v^{1}, \\
0 & =\left(J_{x} L(x, \lambda)+\nabla_{y} g(x, x) D_{\lambda}^{-1} D_{h} J h(x)\right) v^{1} .
\end{aligned}
$$

Thus, nonsingularity of $J \Psi(x, \lambda)$ is equivalent to the nonsingularity of the matrix $J_{x} L(x, \lambda)+\nabla_{y} g(x, x) D_{\lambda}^{-1} D_{h} J h(x)$.

We remark that the assumption $h(x) \neq 0$ in Proposition 4.3 is mandatory to exploit the reduced matrix given in the proposition. In fact, if $h_{i}(x)=0$ for even one single element, the matrix $D_{\lambda}$ becomes singular.

The first part of the following result was proved in [21, Corollary 2]. The second one follows from the first one with Proposition 4.1.

Proposition 4.4 Let (A1++) hold. Then,

(a) $J H(x, \lambda, w)$ is nonsingular for all $(x, \lambda, w) \in \mathbb{R}^{n} \times \mathbb{R}_{++}^{m} \times \mathbb{R}_{++}^{m}$, i.e., (F6) holds;

(b) $J \Psi(x, \lambda)$ is nonsingular for all $(x, \lambda) \in \mathbb{R}^{n} \times \mathbb{R}_{++}^{m}$ with $h(x)<0$, i.e., (F7) holds.

For checking nonsingularity of $J H(x, \lambda, w)$ for all $(\lambda, w) \in \mathbb{R}_{++}^{m} \times \mathbb{R}_{++}^{m}$, it would be nicer to have a condition that is independent of the choice of $(\lambda, w)$. In this perspective, if (A4) holds (meaning that we have linear constraints with variable right-hand side), we obtain exactly what we want, that is, (A1) does not involve the variables $\lambda$, because now, the matrices $J_{x} L(x)$ and $M(x)$ depend exclusively on $x$. This also means that $(\mathrm{A} 1),(\mathrm{A} 1+)$ and $(\mathrm{A} 1++)$ coincide. Moreover, under $(\mathrm{A} 4)$, we derive sharper nonsingularity results.

Now, we state our main theorem, a necessary and sufficient condition for the matrix $J H(x, \lambda, w)$ to be nonsingular for all $\lambda, w \in \mathbb{R}_{++}^{m}$. This theorem and the following corollary are a nontrivial and fundamental generalization of the results of [21], where only sufficiency was proved.

Theorem 4.1 Let $x \in \mathbb{R}^{n}$ be given, and let (A4) hold. Then, $J H(x, \lambda, w)$ is nonsingular for all $\lambda, w \in \mathbb{R}_{++}^{m}$, if and only if (A5) holds, i.e., for some $J \subseteq\{1, \ldots, m\}$, 
$d_{J}:=\operatorname{det}\left(\begin{array}{cc}J_{x} F(x) & \nabla_{y} g_{\bullet} J \\ -J h_{J \bullet}(x) & 0\end{array}\right) \neq 0$ and either $d_{J} \geq 0$ for all $J \subseteq\{1, \ldots, m\}$, or $d_{J} \leq 0$ for all $J \subseteq\{1, \ldots, m\}$.

Proof We have $\operatorname{det}(J H(x, \lambda, w))=\operatorname{det}\left(\begin{array}{ccc}J_{x} F(x) & \nabla_{y} g & 0 \\ J h(x) & 0 & I \\ 0 & \operatorname{diag}(w) & \operatorname{diag}(\lambda)\end{array}\right)$.

Developing this determinant by the last $m$ rows, we obtain a sum over the index sets $J \subseteq\{1, \ldots, m\}$, where every addend contains products of $\lambda_{i}, i \in J$ and $w_{i}$, $i \in J^{c}:=\{1, \ldots, m\} \backslash J$. For each $i \in J$, we drop the corresponding columns of the last block. For each $\tilde{i} \in J^{c}$, we drop the corresponding column $\nabla_{y} g_{\bullet} \tilde{i}$ in the second block and, since we also drop the row with $\lambda_{\tilde{i}}$, we can develop the remaining matrix after the corresponding column $\tilde{i}$ in the middle right block. This results in dropping the row $J h_{\tilde{i}}(x)$. Further, note that in the determinant formula, we get for every $i \in J$ a positive sign, and for every $i \in J^{c}$ we get one negative and one positive sign for the two developments. Thus, we obtain for the determinant of $J H(x, \lambda, w)$

$$
\begin{aligned}
& \operatorname{det}(J H(x, \lambda, w)) \\
& =\sum_{J \subseteq\{1, \ldots, m\}}(-1)^{\left|J^{c}\right|}\left(\prod_{i \in J^{c}} w_{i}\right)\left(\prod_{i \in J} \lambda_{i}\right) \operatorname{det}\left(\begin{array}{cc}
J_{x} F(x) & \nabla_{y} g \bullet J \\
J h_{J \bullet}(x) & 0
\end{array}\right) \\
& =\sum_{J \subseteq\{1, \ldots, m\}}(-1)^{\left|J^{c}\right|}(-1)^{|J|}\left(\prod_{i \in J^{c}} w_{i}\right)\left(\prod_{i \in J} \lambda_{i}\right) \operatorname{det}\left(\begin{array}{cc}
J_{x} F(x) & \nabla_{y} g \bullet J \\
-J h_{J}(x) & 0
\end{array}\right) \\
& =(-1)^{m} \sum_{J \subseteq\{1, \ldots, m\}}\left(\prod_{i \in J^{c}} w_{i}\right)\left(\prod_{i \in J} \lambda_{i}\right) d_{J} .
\end{aligned}
$$

If $d_{J} \neq 0$ for some $J \subseteq\{1, \ldots, m\}$, and, either $d_{J} \geq 0$ for all $J \subseteq\{1, \ldots, m\}$, or $d_{J} \leq 0$ for all $J \subseteq\{1, \ldots, m\}$, i.e., if (A5) holds, we have $\operatorname{det}(J H(x, \lambda, w)) \neq 0$ for all $\lambda, w \in \mathbb{R}_{++}^{m}$, and hence, $J H(x, \lambda, w)$ is nonsingular.

Now, assume (A5) does not hold. Then, we either have $d_{J}=0$ for all $J \subseteq\{1, \ldots, m\}$, which immediately implies that $\operatorname{det}(J H(x, \lambda, w))=0$, or we have index sets $J_{1}, J_{2} \subseteq\{1, \ldots, m\}$ such that

$$
d_{J_{1}}>0>d_{J_{2}}
$$

In this case, let us define

$$
d_{\max }:=\max _{J \subseteq\{1, \ldots, m\}}\left|d_{J}\right| \quad \text { and } \quad d_{\min }:=\min _{J \subseteq\{1, \ldots, m\},\left|d_{J}\right| \neq 0}\left|d_{J}\right| .
$$

Now we set

$$
\lambda_{i}=\left\{\begin{array}{ll}
2^{m}, & \text { if } i \in J_{1} \cap J_{2}, \\
z, & \text { if } i \in J_{1} \backslash J_{2}, \\
\frac{1}{z}, & \text { if } i \in J_{2} \backslash J_{1}, \\
\frac{d_{\min }}{d_{\max }}, & \text { else, }
\end{array} \quad \text { and } \quad w_{i}= \begin{cases}2^{m}, & \text { if } i \in J_{1}^{c} \cap J_{2}^{c}, \\
z, & \text { if } i \in J_{1}^{c} \backslash J_{2}^{c}, \\
\frac{1}{z}, & \text { if } i \in J_{2}^{c} \backslash J_{1}^{c}, \\
\frac{d_{\min }}{d_{\max }}, & \text { else. }\end{cases}\right.
$$


With these settings $f: \mathbb{R}_{++} \rightarrow \mathbb{R}$,

$$
f(z):=\sum_{J \subseteq\{1, \ldots, m\}}\left(\prod_{i \in J^{c}} w_{i}\right)\left(\prod_{i \in J} \lambda_{i}\right) d_{J}
$$

is a continuous function. To analyze its behavior for $z \rightarrow+\infty$, we have to consider the coefficients for the terms with the largest power of $z$, which is $\left|J_{1} \backslash J_{2}\right|+\left|J_{1}^{c} \backslash J_{2}^{c}\right|$. Since $d_{J_{1}}>0$, we obtain for the index set $J_{1}$ the positive addend

$$
z^{\left|J_{1} \backslash J_{2}\right|+\left|J_{1}^{c} \backslash J_{2}^{c}\right|}\left(2^{m}\right)^{\left|J_{1} \cap J_{2}\right|+\left|J_{1}^{c} \cap J_{2}^{c}\right|} d_{J_{1}}
$$

The remaining index sets $\tilde{J} \neq J_{1}$ leading to the largest power of $z$ must satisfy $J_{1} \backslash J_{2} \subseteq \tilde{J}$ and $J_{1}^{c} \backslash J_{2}^{c} \subseteq \tilde{J}$. By construction, we have natural numbers $\alpha \leq\left|J_{1} \cap J_{2}\right|+\left|J_{1}^{c} \cap J_{2}^{c}\right|-1$ and $\beta \geq 1$, such that the corresponding addend satisfies

$$
\begin{aligned}
& z^{\left|J_{1} \backslash J_{2}\right|+\left|J_{1}^{c} \backslash J_{2}^{c}\right|}\left(2^{m}\right)^{\alpha}\left(\frac{d_{\min }}{d_{\max }}\right)^{\beta} d_{\tilde{J}} \\
& \quad \leq z^{\left|J_{1} \backslash J_{2}\right|+\left|J_{1}^{c} \backslash J_{2}^{c}\right|}\left(2^{m}\right)^{\left|J_{1} \cap J_{2}\right|+\left|J_{1}^{c} \cap J_{2}^{c}\right|-1} \frac{d_{\min }}{d_{\max }}\left|d_{\tilde{J}}\right| \\
& \quad \leq z^{\left|J_{1} \backslash J_{2}\right|+\left|J_{1}^{c} \backslash J_{2}^{c}\right|}\left(2^{m}\right)^{\left|J_{1} \cap J_{2}\right|+\left|J_{1}^{c} \cap J_{2}^{c}\right|-1} d_{J_{1}}
\end{aligned}
$$

Since the total number of possible index sets $\tilde{J} \neq J_{1}$ is not greater than $2^{m}-1$, and

$$
\left(2^{m}-1\right)\left(2^{m}\right)^{\left|J_{1} \cap J_{2}\right|+\left|J_{1}^{c} \cap J_{2}^{c}\right|-1} d_{J_{1}}<\left(2^{m}\right)^{\left|J_{1} \cap J_{2}\right|+\left|J_{1}^{c} \cap J_{2}^{c}\right|} d_{J_{1}},
$$

the coefficient of $z^{\left|J_{1} \backslash J_{2}\right|+\left|J_{1}^{c} \backslash J_{2}^{c}\right|}$ is positive. Thus, we have

$$
\lim _{z \rightarrow \infty} f(z)=+\infty
$$

Using analogous arguments for the index set $J_{2}$ with $d_{J_{2}}<0$, we can show that the coefficient for the largest power of $\frac{1}{z}$ is negative and hence

$$
\lim _{z \rightarrow 0} f(z)=-\infty
$$

Now, by continuity of $f$, it has a zero. Thus, we can find $\lambda, w \in \mathbb{R}_{++}^{m}$, such that $\operatorname{det}(J H(x, \lambda, w))=0$, and hence, $J H(x, \lambda, w)$ is singular, which completes the proof.

This theorem shows that under (A4) the nonsingularity condition (F6) is equivalent to (A5) holding for all $x \in \mathbb{R}^{n}$. Next, we present a new corollary showing that under (A4) and nonsingularity of $J_{x} F(x),(\mathrm{A} 1)$ is necessary and sufficient for (F6).

Corollary 4.1 Let $x \in \mathbb{R}^{n}$ be given, (A4) hold, and $J_{x} F(x)$ be nonsingular. Then, the matrix $J H(x, \lambda, w)$ is nonsingular for all $\lambda, w \in \mathbb{R}_{++}^{m}$, if and only if $M(x)$ is a $P_{0}$ matrix. 
Proof By Theorem 4.1, $J H(x, \lambda, w)$ being nonsingular means that for some $J \subseteq\{1, \ldots, m\}$ we have $d_{J}=\operatorname{det}\left(\begin{array}{cc}J_{x} F(x) & \nabla_{y} g_{\bullet} J \\ -J h_{J \bullet}(x) & 0\end{array}\right) \neq 0$ and either $d_{J} \geq 0$ for all $J \subseteq\{1, \ldots, m\}$ or $d_{J} \leq 0$ for all $J \subseteq\{1, \ldots, m\}$. By the assumption on $J_{x} F(x)$, we have $d_{\emptyset}=\operatorname{det} J_{x} F(x) \neq 0$. Further, for $J \neq \emptyset$ we can use the determinant formula for block matrices to obtain

$$
d_{J}=\operatorname{det}\left(J_{x} F(x)\right) \operatorname{det}\left(J_{J} \bullet(x) J_{x} F(x)^{-1} \nabla_{y} g_{\bullet J}\right)=d_{\emptyset} \operatorname{det}\left(M_{J J}(x)\right) .
$$

Thus, the nonsingularity of $J H$ is equivalent to requiring that

$$
\operatorname{det}\left(M_{J J}(x)\right) \geq 0, \quad \forall J \subseteq\{1, \ldots, m\}, \quad J \neq \varnothing .
$$

This coincides with the fact that $M(x)$ is a $P_{0}$ matrix, and proves the equivalence.

Let us present an example that illustrates the applicability of the corollary, in particular, if we have a linear function $F$ yielding that $M(x)$ becomes independent of $x$.

Example 4.1 Define $F: \mathbb{R}^{2} \rightarrow \mathbb{R}^{2}$ and $g: \mathbb{R}^{2} \times \mathbb{R}^{2} \rightarrow \mathbb{R}^{2}$ by

$$
F(x)=\left(\begin{array}{l}
2 x_{2}-1 \\
2 x_{1}-1
\end{array}\right) \quad \text { and } \quad g(y, x)=\left(\begin{array}{l}
x_{1}+2 y_{2}-1 \\
x_{2}+2 y_{1}-1
\end{array}\right)
$$

The QVI has the unique solution $\left(\frac{1}{3}, \frac{1}{3}\right)$. We have (A4), and $J_{x} F=\left(\begin{array}{ll}0 & 2 \\ 2 & 0\end{array}\right)$ is nonsingular. But, the matrix

$$
M=M(x)=\left(\begin{array}{ll}
1 & 2 \\
2 & 1
\end{array}\right)\left(\begin{array}{ll}
0 & \frac{1}{2} \\
\frac{1}{2} & 0
\end{array}\right)\left(\begin{array}{ll}
0 & 2 \\
2 & 0
\end{array}\right)=\left(\begin{array}{ll}
1 & 2 \\
2 & 1
\end{array}\right)
$$

is not a $P_{0}$ matrix for any $x \in \mathbb{R}^{n}$. Hence, by Corollary $4.1, J H(x, \lambda, w)$ is for all $x \in \mathbb{R}^{n}$ singular for some $\lambda, w \in \mathbb{R}_{++}^{m}$. For example, one can set $\lambda_{1}=\lambda_{2}=w_{1}=w_{2}$.

Let us further mention that $M$ is semimonotone, and hence, (A2) is satisfied. Thus, Proposition 3.2 yields the stationarity result (F2) in this case, where nonsingularity of $J H(x, \lambda, w)$ is violated.

From Proposition 4.1, we obtain the following corollaries for nonsingularity of $J \Psi(x, \lambda)$ :

Corollary 4.2 Let $x \in \mathbb{R}^{n}$ with $h(x)<0$ be given, and let (A4) hold. Then, the matrix $J \Psi(x, \lambda)$ is nonsingular for all $\lambda \in \mathbb{R}_{++}^{m}$, if and only if (A5) holds.

Corollary 4.3 Let $x \in \mathbb{R}^{n}$ with $h(x)<0$ be given, (A4) hold and $J_{x} F(x)$ be nonsingular. Then, the matrix $J \Psi(x, \lambda)$ is nonsingular for all $\lambda \in \mathbb{R}_{++}^{m}$, if and only if $M(x)$ is a $P_{0}$ matrix. 


\section{QVIs with Box Constraints}

Now, we discuss the case of QVIs with additional box constraints, i.e.,

$$
\bar{K}(x):=\left\{y \in[l, u] \subseteq \mathbb{R}^{n}: g(y, x) \leq 0\right\}
$$

The KKT conditions become

$$
\begin{aligned}
& L(x, \lambda):=F(x)+\lambda^{1}-\lambda^{2}+\nabla_{y} g(x, x) \lambda^{3}=0, \\
& \lambda_{i}^{1} \geq 0, x_{i}-u_{i} \leq 0, \lambda_{i}^{1}\left(x_{i}-u_{i}\right)=0 \quad \forall i=1, \ldots, n, \\
& \lambda_{i}^{2} \geq 0, l_{i}-x_{i} \leq 0, \lambda_{i}^{2}\left(l_{i}-x_{i}\right)=0 \quad \forall i=1, \ldots, n, \\
& \lambda_{i}^{3} \geq 0, g_{i}(x, x) \leq 0, \lambda_{i}^{3} g_{i}(x, x)=0 \quad \forall i=1, \ldots, m .
\end{aligned}
$$

The constrained nonlinear system in the box constrained case is to solve

$$
H(x, \lambda, w):=\left(\begin{array}{c}
L(x, \lambda) \\
x-u+w^{1} \\
-x+l+w^{2} \\
h(x)+w^{3} \\
\lambda^{1} \circ w^{1} \\
\lambda^{2} \circ w^{2} \\
\lambda^{3} \circ w^{3}
\end{array}\right)=0 \quad(x, \lambda, w) \in \mathbb{R}^{n} \times \mathbb{R}_{+}^{m} \times \mathbb{R}_{+}^{m}
$$

We show that the presence of the box constraints can be exploited in order to obtain the facts (F1)-(F7) under weakened assumptions. First, we will give some sufficient conditions for (A2) that entails (F2) and (F3).

Proposition 5.1 Let a QVI with box constraints be given and all entries in $J h(x)$ be nonnegative. If $J_{x} L(x, \lambda)$ is a positive diagonal matrix, the matrix $M(x, \lambda)$ is semimonotone, i.e., (A2) is satisfied.

Proof To show that

$$
\begin{aligned}
& M(x, \lambda)=\left(\begin{array}{c}
I \\
-I \\
J h(x)
\end{array}\right) J_{x} L(x, \lambda)^{-1}\left(I-I \nabla_{y} g(x, x)\right) \\
& \quad=\left(\begin{array}{ccc}
J_{x} L(x, \lambda)^{-1} & -J_{x} L(x, \lambda)^{-1} & J_{x} L(x, \lambda)^{-1} \nabla_{y} g(x, x) \\
-J_{x} L(x, \lambda)^{-1} & J_{x} L(x, \lambda)^{-1} & -J_{x} L(x, \lambda)^{-1} \nabla_{y} g(x, x) \\
J h(x) J_{x} L(x, \lambda)^{-1} & -J h(x) J_{x} L(x, \lambda)^{-1} & J h(x) J_{x} L(x, \lambda)^{-1} \nabla_{y} g(x, x)
\end{array}\right)
\end{aligned}
$$

is semimonotone, let a vector $v=\left(v^{1}, v^{2}, v^{3}\right) \in \mathbb{R}_{+}^{n+n+m} \backslash\{0\}$ be given.

First, assume $v_{j}^{1}>0$ for some $j \in\{1, \ldots, n\}$. Then, we must either have $M_{j \bullet}(x, \lambda) v \geq 0$ (and hence $M(x, \lambda)$ is semimonotone), or we get from the $j$ th row $v_{j}^{2}>0$, since $J_{x} L(x, \lambda)^{-1}$ is a positive diagonal matrix and with $J h(x)$ also all entries in $\nabla_{y} g(x, x)$ are nonnegative. Since the $j$ th row is the negative of the $(n+j)$ th row, 
either $M_{j \bullet}(x, \lambda) v \geq 0$ or $M_{(n+j) \bullet}(x, \lambda) v \geq 0$ must hold, and hence, $M(x, \lambda)$ must be semimonotone.

Now assume $v^{1}=0$, and define the set

$$
J:=\left\{j \in\{1, \ldots, n\}: v_{j}^{2}>0\right\}
$$

If $J=\emptyset$, we have $v^{2}=0$. Since the matrix $J h(x) J_{x} L(x, \lambda)^{-1} \nabla_{y} g(x, x)$ has only nonnegative entries, it is semimonotone, and hence, $M(x, \lambda)$ is semimontone. Thus, assume $J \neq \emptyset$. We are done, if one of the components

$$
J_{x} L_{J \bullet}(x, \lambda)^{-1} v^{2}-J_{x} L_{J \bullet}(x, \lambda)^{-1} \nabla_{y} g(x, x) v^{3}
$$

is nonnegative. Hence, assume they are all negative. For $J \neq \varnothing$ the fact that $J_{x} L(x, \lambda)^{-1}$ is a positive diagonal matrix immediately implies that at least one component of $v^{3}$ must be positive. Since all entries in $J_{x} L(x, \lambda)^{-1} \nabla_{y} g(x, x) v^{3}$ are nonnegative, we have

$$
J_{x} L(x, \lambda)^{-1} v^{2}-J_{x} L(x, \lambda)^{-1} \nabla_{y} g(x, x) v^{3} \leq 0
$$

Multiplying with the nonpositive matrix $-J h(x)$ results in

$$
-J h(x) J_{x} L(x, \lambda)^{-1} v^{2}+J h(x) J_{x} L(x, \lambda)^{-1} \nabla_{y} g(x, x) v^{3} \geq 0
$$

Since this corresponds to the last $m$ rows of $M(x, \lambda)$, the matrix must be semimonotone since one of the components of $v^{3}$ is positive.

The assumption (A3) is restrictive in the case without box constraints and does not hold, for example, if $M(x, \lambda)$ consists of two rows, where one is the negative of the other. A different condition can be obtained for QVIs with additional box constraints. Let us denote

$$
\tilde{g}(y, x):=\left(\begin{array}{c}
y-u \\
l-y \\
g(y, x)
\end{array}\right) .
$$

Here, we can use a positive linear independence assumption, rather than (A3), to obtain a constrained stationarity result.

Theorem 5.1 Let a QVI with box constraints be given. Assume that for all $(x, \lambda) \in \mathbb{R}^{n} \times \mathbb{R}_{+}^{m}$, the matrix $J_{x} L(x, \lambda)$ is nonsingular and that the matrix $M(x, \lambda)=$ $J \widetilde{h}(x) J_{x} L(x, \lambda)^{-1} \nabla_{y} \widetilde{g}(x, x)$ is positive semidefinite. Further, assume the gradients $\nabla \widetilde{h}_{j}(x)$ for $j \in J:=\left\{j \in\{1, \ldots, m\}: \widetilde{h}_{j}(x)>0\right\}$ are positive linear independent. Then, every constrained stationary point of the merit function $\frac{1}{2}\|H(x, \lambda, w)\|^{2}$ subject to $\lambda \geq 0$ and $w \geq 0$ is a zero of $H$, i.e., (F5) is true. 
Proof First, we can follow the lines of the proof of Theorem 3.2 to obtain (8) and thus to exclude the case (a), which was $\lambda_{i}>0$ and $w_{i}>0$, through the assumed positive semidefiniteness of $M(x, \lambda)$. Thus, we must have $\lambda \circ w=0$. Now, we inspect the constrained stationarity conditions for the box constraints. Then, we obtain for every $i=1, \ldots, n$ :

$$
\begin{aligned}
& \lambda_{i}^{1}>0 \quad \Rightarrow \quad L_{i}(x, \lambda)+\left(w_{i}^{1}\right)^{2} \lambda_{i}^{1}=0 \quad \Rightarrow \quad L_{i}(x, \lambda)=0, \\
& \lambda_{i}^{2}>0 \quad \Rightarrow-L_{i}(x, \lambda)+\left(w_{i}^{2}\right)^{2} \lambda_{i}^{2}=0 \quad \Rightarrow \quad L_{i}(x, \lambda)=0, \\
& \lambda_{i}^{1}=0 \quad \Rightarrow \quad L_{i}(x, \lambda)+\left(w_{i}^{1}\right)^{2} \lambda_{i}^{1} \geq 0 \quad \Rightarrow \quad L_{i}(x, \lambda) \geq 0 . \\
& \lambda_{i}^{2}=0 \quad \Rightarrow-L_{i}(x, \lambda)+\left(w_{i}^{2}\right)^{2} \lambda_{i}^{2} \geq 0 \quad \Rightarrow-L_{i}(x, \lambda) \geq 0,
\end{aligned}
$$

If $\lambda_{i}^{1}>0$ or $\lambda_{i}^{2}>0$, the first two lines imply $L_{i}(x, \lambda)=0$. If both $\lambda_{i}^{1}=0$ and $\lambda_{i}^{2}=0$, the lines three and four imply again $L_{i}(x, \lambda)=0$. Since this holds for all $i=1, \ldots, n$, we have $L(x, \lambda)=0$.

It remains to show that $\widetilde{h}(x)+w=0$. From the constrained stationarity conditions, we get with $\lambda \circ w=0$ :

$$
\begin{aligned}
& w_{i}>0 \quad \Rightarrow \widetilde{h}_{i}(x)+w_{i}+w_{i} \lambda_{i}^{2}=0 \quad \Rightarrow \widetilde{h}_{i}(x)+w_{i}=0 \\
& w_{i}=0 \quad \Rightarrow \widetilde{h}_{i}(x)+w_{i}+w_{i} \lambda_{i}^{2} \geq 0 \quad \Rightarrow \quad \widetilde{h}_{i}(x) \geq 0
\end{aligned}
$$

This together with $L(x, \lambda)=0$ and $J:=\left\{j \in\{1, \ldots, m\}: \widetilde{h}_{j}(x)>0\right\}$ implies that the remaining stationarity condition yields

$$
0=\nabla_{x} L(x, \lambda) L(x, \lambda)+\nabla \tilde{h}(x)(\widetilde{h}(x)+w)=\nabla \tilde{h}_{\bullet J}(x) \tilde{h}_{J}(x)
$$

Since $\widetilde{h}_{J}(x)>0$ and the gradients $\nabla \widetilde{h}_{j}(x), j \in J$ are positively linearly independent, we must have $J=\emptyset$, which means $\widetilde{h}(x)+w=0$, and completes the proof.

In the box constrained case, the Jacobian $J H(x, \lambda, w)$ is given by

$\left(\begin{array}{ccccccc}J_{x} L(x) & I & -I & \nabla_{y} g(x, x) & 0 & 0 & 0 \\ I & 0 & 0 & 0 & I & 0 & 0 \\ -I & 0 & 0 & 0 & 0 & I & 0 \\ J h(x) & 0 & 0 & 0 & 0 & 0 & I \\ 0 & \operatorname{diag}\left(w^{1}\right) & 0 & 0 & \operatorname{diag}\left(\lambda^{1}\right) & 0 & 0 \\ 0 & 0 & \operatorname{diag}\left(w^{2}\right) & 0 & 0 & \operatorname{diag}\left(\lambda^{2}\right) & 0 \\ 0 & 0 & 0 & \operatorname{diag}\left(w^{3}\right) & 0 & 0 & \operatorname{diag}\left(\lambda^{3}\right)\end{array}\right)$.

Let $\lambda, w \in \mathbb{R}_{++}^{2 n+m}$ be given, and let us define the matrix

$$
\widetilde{J_{x} L}(x, \lambda, w):=J_{x} L(x)+\operatorname{diag}\left(\lambda^{1} \circ\left(w^{1}\right)^{-1}\right)+\operatorname{diag}\left(\lambda^{2} \circ\left(w^{2}\right)^{-1}\right) .
$$


It is not difficult to see that $J H(x, \lambda, w)$ is nonsingular, if and only if

$$
\left(\begin{array}{ccc}
J_{x} L(x, \lambda, w) & \nabla_{y} g(x, x) & 0 \\
J h(x) & 0 & I \\
0 & \operatorname{diag}\left(w^{3}\right) & \operatorname{diag}\left(\lambda^{3}\right)
\end{array}\right)
$$

is nonsingular. In this setting, we can replace the nonsingularity of $J_{x} L(x, \lambda)$ from $(\mathrm{A} 1++)$ by positive semidefiniteness of $J_{x} L(x, \lambda)$ in Proposition 4.4 and in Corollary 4.1. In fact, in this case $\widetilde{J_{x} L}(x, \lambda, w)$ becomes nonsingular for all $\lambda, w \in \mathbb{R}_{++}^{m}$, and the developments follow as in the case without box constraints. We observe that this also holds, if we have either a lower or an upper bound for each variable, and we do not need both of them. Defining

$$
\tilde{M}(x):=J h(x) \widetilde{J_{x} L}(x, \lambda, w)^{-1} \nabla_{y} g(x, x),
$$

we get the following results.

Corollary 5.1 Assume box constraints. Let the matrix $J_{x} L(x, \lambda)$ be positive semidefinite and the matrix $\widetilde{M}(x)$ be a $P_{0}$ matrix for all $(x, \lambda) \in \mathbb{R}^{n} \times \mathbb{R}_{++}^{m}$. Then,

(a) $J H(x, \lambda, w)$ is nonsingular for all $(x, \lambda, w) \in \mathbb{R}^{n} \times \mathbb{R}_{++}^{m} \times \mathbb{R}_{++}^{m}$, i.e., (F6) holds;

(b) $J \Psi(x, \lambda)$ is nonsingular for all $(x, \lambda) \in \mathbb{R}^{n} \times \mathbb{R}_{++}^{m}$ with $h(x)<0$, i.e., (F7) holds.

Corollary 5.2 Assume we have box constraints, (A4) holds, and $J_{x} F(x)$ is positive semidefinite for all $x \in \mathbb{R}^{n}$. Then,

(a) (F6) is equivalent to $\tilde{M}(x)$ being a $P_{0}$ matrix;

(b) (F7) is equivalent to $\widetilde{M}(x)$ being a $P_{0}$ matrix for all $x \in \mathbb{R}^{n}$ with $h(x)<0$.

Moreover, if $J_{x} F(x)$ is positive definite for all $x \in \mathbb{R}^{n}$, then

(c) $\tilde{M}(x)$ is a $P_{0}$ matrix if and only if $M(x)$ is a $P_{0}$ matrix.

Proof Point (c) is due to point (a) and Corollary 4.1.

\section{GNEPS}

It was first observed in [7] that under mild assumptions a GNEP can be reformulated as an equivalent QVI. Hence, we can obtain GNEP results by applying the QVI results from the last sections. We will not state all of them here, but we will focus on special cases, where we can obtain new results.

We consider GNEPs with linear constraints and quadratic cost functions. Let matrices $Q_{\nu} \in \mathbb{R}^{n_{\nu} \times n_{\nu}}$ and $A_{\nu \mu} \in \mathbb{R}^{m_{\nu} \times n_{\nu}}$, and vectors $c^{\nu} \in \mathbb{R}^{n_{\nu}}$ and $b^{\nu} \in \mathbb{R}^{m_{\nu}}$ be given. Further, $X_{v}$ is a closed convex subset of $\mathbb{R}^{n_{v}}$. Each player $v=1, \ldots, N$ tries to find $x^{v} \in \mathbb{R}^{n_{v}}$ to solve the problem

$$
\min _{x^{\nu}} \frac{1}{2}\left(x^{\nu}\right)^{\top} Q_{\nu} x^{\nu}+\left(c^{\nu}\right)^{\top} x^{\nu} \quad \text { s.t. } \quad \sum_{\mu=1}^{N} A_{\nu \mu} x^{\mu} \leq b^{\nu}, \quad x^{\nu} \in X_{\nu} .
$$


Notice that the GNEPs considered here have objective functions that depend only on private variables, while the constraints may couple all the variables. We underline that the coupling constraints are not necessarily shared by all the players, and therefore, these GNEPs are, in general, no generalized potential games as defined in $[37,38]$. The QVI reformulation of the GNEP is made up by

$$
F(x)=\left(\begin{array}{ccc}
Q_{1} & & \\
& \ddots & \\
& & Q_{N}
\end{array}\right) x+\left(\begin{array}{c}
c^{1} \\
\vdots \\
c^{N}
\end{array}\right), g(y, x)=\left(\begin{array}{c}
A_{11} y^{1}+\sum_{\mu \neq 1} A_{1 \mu} x^{\mu}-b^{1} \\
\vdots \\
A_{N N} y^{N}+\sum_{\mu \neq N} A_{N \mu} x^{\mu}-b^{N}
\end{array}\right) .
$$

By the linearity of the constraints, we have (A4).

For completeness, let us first mention that the case of linear cost functions, i.e., if we have $Q_{v}=0$ for all $v=1, \ldots, N$, is discussed in [32]. In particular, we have in [32, Theorem 4.1] the following result:

Lemma 6.1 Let $Q_{v}=0$ and $X_{v}=\mathbb{R}^{n_{v}}$ for all $v \in\{1, \ldots, N\}$. Assume that $\operatorname{det}\left(\nabla_{y} g_{\bullet J} J h_{J_{\bullet}}\right) \geq 0$ for all $J \subseteq\{1, \ldots, m\}$ with $|J|=n$, and that the determinant is positive for one of these sets. Then, $J H(\lambda, w)$ is nonsingular for all $\lambda, w \in \mathbb{R}_{++}^{m}$.

Exploiting Theorem 4.1, we can extend Lemma 6.1 and get necessary and sufficient conditions for the nonsingularity.

Corollary 6.1 Let $Q_{v}=0$ and $X_{v}=\mathbb{R}^{n_{v}}$ for all $v \in\{1, \ldots, N\} . J H(\lambda, w)$ is nonsingular for all $\lambda, w \in \mathbb{R}_{++}^{m}$, if and only if all determinants $\operatorname{det}\left(\nabla_{y} g_{\bullet J} J h_{J_{\bullet}}\right)$ with $J \subseteq\{1, \ldots, m\}$ and $|J|=n$, are either all nonnegative or all nonpositive, and at least one of them is not 0 .

Proof By Theorem 4.1, we consider the signs of $d_{J}:=\operatorname{det}\left(\begin{array}{cc}0 & \nabla_{y} g \bullet J \\ -J h_{J} & 0\end{array}\right)$. We have $d_{J}=0$ for every $J \subseteq\{1, \ldots, m\}$ with $|J| \neq n$. For $|J|=n$ we have

$$
\begin{aligned}
d_{J} & =(-1)^{n} \operatorname{det}\left(\begin{array}{cc}
-J h_{J} & 0 \\
0 & \nabla_{y} g_{\bullet J}
\end{array}\right)=(-1)^{2 n} \operatorname{det}\left(J h_{J \bullet}\right) \operatorname{det}\left(\nabla_{y} g_{\bullet}\right) \\
& =\operatorname{det}\left(\nabla_{y} g_{\bullet J} J h_{J \bullet}\right) .
\end{aligned}
$$

Now, the assertion is a consequence of Theorem 4.1 .

In the next example, the matrix $J H(x, \lambda, w)$ is always singular.

Example 6.1 Consider the linear 2-player GNEP

$$
\begin{array}{lll}
\min -x^{1} & \text { s.t. } & x^{1}+x^{2} \leq 1 \\
\min -x^{2} & \text { s.t. } & x^{1}+x^{2} \leq 1
\end{array}
$$

From Lemma 6.1, we get that $J H(x, \lambda, w)$ is singular for some $\lambda, w \in \mathbb{R}_{++}^{m}$, since for $J=\{1,2\}$ we get $\operatorname{det}\left(\nabla_{y} g_{\bullet} J h_{J}\right)=\operatorname{det}\left(\left(\begin{array}{ll}1 & 0 \\ 0 & 1\end{array}\right)\left(\begin{array}{ll}1 & 1 \\ 1 & 1\end{array}\right)\right)=0$. But even more, 
in this example $J H(x, \lambda, w)$ is singular for all $\lambda, w \in \mathbb{R}_{++}^{m}$, since

$$
\begin{array}{r}
\operatorname{det}(J H(x, \lambda, w))=\lambda_{1} \lambda_{2} \operatorname{det}\left(\begin{array}{cccc}
0 & 0 & 1 & 0 \\
0 & 0 & 0 & 1 \\
-1 & -1 & 0 & 0 \\
-1 & -1 & 0 & 0
\end{array}\right)+\lambda_{1} w_{2} \operatorname{det}\left(\begin{array}{ccc}
0 & 0 & 1 \\
0 & 0 & 0 \\
-1 & -1 & 0
\end{array}\right) \\
+\lambda_{2} w_{1} \operatorname{det}\left(\begin{array}{ccc}
0 & 0 & 0 \\
0 & 0 & 1 \\
-1 & -1 & 0
\end{array}\right)+w_{1} w_{2} \operatorname{det}\left(\begin{array}{cc}
-1 & -1 \\
-1 & -1
\end{array}\right)=0 .
\end{array}
$$

Next, we consider the more general quadratic setting. However, we restrict ourselves to GNEPs, where all players have the same number of variables, i.e., we assume $n_{1}=\ldots=n_{N}$. For some special structures, we get the facts $(\mathrm{F} 1)-(\mathrm{F} 7)$.

Theorem 6.1 Let $n_{1}=\ldots=n_{N}, Q_{v}=\beta_{v} T$ with $\beta_{v} \in \mathbb{R}_{++}$and a positive definite matrix $T \in \mathbb{R}^{n_{1} \times n_{1}}$, and $X_{v}=\mathbb{R}^{n_{1}}$ for all $v \in\{1, \ldots, N\}$. Assume $A_{v \mu}=\alpha_{\mu}\left(B_{v}+E_{v \mu}\right)$ with $\alpha_{\mu} \in \mathbb{R}$ and $B_{v}, E_{v \mu} \in \mathbb{R}^{m_{v} \times n_{1}}, E_{v v}=0$, for all $\mu, v \in\{1, \ldots, N\}$.

1. Let all entries of either $E_{v \mu} T^{-1} B_{\mu}$ or $\left(B_{v}+E_{v \mu}\right) T^{-1} B_{\mu}$ be nonnegative for all $\mu, v \in\{1, \ldots, N\}$. Then, $M$ is a semimonotone matrix, i.e., (A2) holds, implying (F2) and (F3).

2. Let $E_{v \mu}=0$ for all $\mu, v \in\{1, \ldots, N\}$. Then, $M$ is a $P_{0}$-matrix, i.e., (A1) holds, implying (F1)-(F4), (F6) and (F7).

3. Let $E_{v \mu}=0$, let $B_{v}$ be a positive matrix, let $T^{-1}$ be a nonnegative matrix with a positive diagonal, and let $\frac{\alpha_{v}^{2}}{\beta_{\nu}}=\frac{\alpha_{\mu}^{2}}{\beta_{\mu}}>0$ for all $\mu, v \in\{1, \ldots, N\}$. Then, $M$ is a positive semidefinite $S$ matrix, i.e., (A3) holds, implying that all the facts (F1)-(F7) are true.

Proof We start with case 2: $J_{x} L(x)=\left(\begin{array}{ccc}\beta_{1} T & & \\ & \ddots & \\ & & \beta_{N} T\end{array}\right)$, is nonsingular, by the assumed positive definiteness of $T$ and since $\beta_{i}>0$ for all $i=1, \ldots, N$. Further, we have

$$
\begin{aligned}
& M=\left(\begin{array}{ccc}
\alpha_{1} B_{1} & \cdots & \alpha_{N} B_{1} \\
\vdots & & \vdots \\
\alpha_{1} B_{N} & \cdots & \alpha_{N} B_{N}
\end{array}\right)\left(\begin{array}{cccc}
\frac{1}{\beta_{1}} T^{-1} & & \\
& \ddots & \\
& & \frac{1}{\beta_{N}} T^{-1}
\end{array}\right)\left(\begin{array}{ccc}
\alpha_{1} B_{1}^{\top} & & \\
& \ddots & \\
& & \alpha_{N} B_{N}^{\top}
\end{array}\right) \\
& =\left(\begin{array}{ccc}
\frac{\alpha_{1}^{2}}{\beta_{1}} B_{1} T^{-1} B_{1}^{\top} & \cdots & \frac{\alpha_{N}^{2}}{\beta_{N}} B_{1} T^{-1} B_{N}^{\top} \\
\vdots & & \vdots \\
\frac{\alpha_{1}^{2}}{\beta_{1}} B_{N} T^{-1} B_{1}^{\top} & \cdots & \frac{\alpha_{N}^{2}}{\beta_{N}} B_{N} T^{-1} B_{N}^{\top}
\end{array}\right) \\
& =\bar{B} T^{-1} \bar{B}^{\top} D \text {, }
\end{aligned}
$$


where $\bar{B}:=\left(\begin{array}{c}B_{1} \\ \vdots \\ B_{N}\end{array}\right)$ and $D:=\left(\begin{array}{ccc}\frac{\alpha_{1}^{2}}{\beta_{1}} I_{m_{1}} & & \\ & \ddots & \\ & & \frac{\alpha_{N}^{2}}{\beta_{N}} I_{m_{N}}\end{array}\right)$. For $J \subseteq\{1, \ldots, m\}$, we have

$$
\operatorname{det}\left(M_{J J}\right)=\operatorname{det}\left(\left(\bar{B} T^{-1} \bar{B}^{\top}\right)_{J J} D_{J J}\right)=\operatorname{det}\left(\left(\bar{B} T^{-1} \bar{B}^{\top}\right)_{J J}\right) \operatorname{det}\left(D_{J J}\right) \geq 0
$$

because $\bar{B} T^{-1} \bar{B}^{\top}$ is positive semidefinite, hence a $P_{0}$ matrix, and $D$ is positive semidefinite and diagonal. Therefore, $M$ is a $P_{0}$ matrix, that is, (A1) is true. As a consequence, (F1) holds by Proposition 3.1 and (F4) by Theorem 3.1. Further, Proposition 4.4 gives (F6) and (F7). Finally, since (A1) implies (A2), we have (F2) from Proposition 3.2 and (F3) from Proposition 3.3.

Now, let us consider case 1: We have

$$
\begin{aligned}
& M=\bar{B} T^{-1} \bar{B}^{\top} D+ \\
& \left(\begin{array}{cccc}
0 & \frac{\alpha_{2}^{2}}{\beta_{2}} E_{12} T^{-1} B_{2}^{\top} & \cdots & \frac{\alpha_{N}^{2}}{\beta_{N}} E_{1 N} T^{-1} B_{N}^{\top} \\
\frac{\alpha_{1}^{2}}{\beta_{1}} E_{21} T^{-1} B_{1}^{\top} & 0 & & \vdots \\
\vdots & & \ddots & \frac{\alpha_{N}^{2}}{\beta_{N}} E_{N-1 N} T^{-1} B_{N}^{\top} \\
\frac{\alpha_{1}^{2}}{\beta_{1}} E_{N} T^{-1} B_{1}^{\top} & \ldots & \frac{\alpha_{N-1}^{2}}{\beta_{N-1}} E_{N N-1} T^{-1} B_{N-1}^{\top} & 0
\end{array}\right) .
\end{aligned}
$$

If the first condition holds, $M$ is the sum of a $P_{0}$ matrix and a nonnegative one; thus, it is semimonotone. If the second condition holds, $M$ is nonnegative and then also semimonotone. In any case, (A2) holds. Now, (F2) follows from Proposition 3.2 and (F3) from Proposition 3.3.

In case 3 , the matrix $M=\bar{B} T^{-1} \bar{B}^{\top} D$, with $D=\frac{\alpha_{1}^{2}}{\beta_{1}} I$, is positive semidefinite. The assumptions on $T^{-1}$ and $B_{v}$ imply that $M z=\frac{\alpha_{1}^{2}}{\beta_{1}} \bar{B} T^{-1} \bar{B}^{\top} z>0$, for any vector $z>0$. Thus, (A3) is true. Fact (F5) comes from Theorem 3.2, while all the others follow as in case 2 , since (A3) implies (A1).

Example 6.2 Let us consider $N$ firms operating in a specific area. Each firm produces its goods in $n_{1}$ spots. Let $x^{\nu} \in \mathbb{R}_{+}^{n_{1}}$ be the quantities of production of the $v$ th firm, and let its cost be given by

$$
\operatorname{cost}_{\nu}\left(x^{\nu}\right):=\left(m^{\nu}\right)^{\top} x^{\nu}+\frac{1}{2} \beta_{v} \sum_{i=1}^{n_{1}}\left(x^{\nu}\right)^{2},
$$

where $m^{v} \in \mathbb{R}_{-}^{n_{1}}$ are the marginal costs and $\beta_{v}>0$. Let $\alpha_{v}>0$ be a number giving the degree of pollution of the $n_{1}$ plants of the $v$ th firm. Any firm $v$ must control the level of pollution in $m_{v}$ different spots of the area:

$$
\alpha_{\nu} B_{v} x^{\nu}+\sum_{\mu \neq v} \alpha_{\mu}\left(B_{v}+E_{v \mu}\right) x^{\mu} \leq b^{v},
$$


where: $B_{v} \in \mathbb{R}_{++}^{m_{v} \times n_{1}}$ represents the absolute pollution impact of the $n_{1}$ spots of production of the $v$ th firm on the $m_{v}$ spots, where the pollution must be controlled by the firm; $E_{v \mu} \in \mathbb{R}^{m_{v} \times n_{1}}$ represents some deviations from $B_{v}$, and $\left(B_{v}+E_{v \mu}\right)$ is the absolute pollution impact of the $\mu$ th firm on the $m_{\nu}$ spots controlled by the $v$ th firm; $b^{v} \in \mathbb{R}_{++}^{m_{v}}$ are the maximum levels of pollution allowed in the $m_{v}$ spots controlled by the $v$ th firm. Any firm $v$ minimizes cost $_{v}$ subject to its pollution constraints.

Referring to Lemma 6.1, we can prove the following assertions.

1. Let all $\left(B_{v}+E_{v \mu}\right) \geq 0$, i.e., the absolute pollution impacts of the firms are nonnegative. Then, (A2) holds and the facts (F2) and (F3) are true.

2. Let $E_{v \mu}=0$ for all $v$ and $\mu$, i.e., the plants of all the firms in any specific spot impact equally on the controlled spots. Then, (A1) holds and facts (F1)-(F4), (F6) and (F7) are true.

3. Let $E_{v \mu}=0$ and $\frac{\alpha_{v}^{2}}{\beta_{v}}=\frac{\alpha_{\mu}^{2}}{\beta_{\mu}}$ for all $v$ and $\mu$, which is, for example, true if the degrees of pollution $\alpha_{v}$ and the parameters $b_{v}$ are the same for all the firms. Then, (A3) holds and all the facts (F1)-(F7) are true.

Now we consider a different framework involving box constraints.

Theorem 6.2 Assume $n_{1}=\ldots=n_{N}, Q_{\nu} \in \mathbb{R}_{+}^{n_{1} \times n_{1}}$ are nonnegative diagonal matrices, $X_{v}=\left[l^{v}, u^{v}\right]$ for all $v=1, \ldots, N$, and $A_{v \mu}=D_{v} \bar{D}_{\mu}+E_{v \mu}$, with diagonal matrices $D_{\nu}, \bar{D}_{\mu} \in \mathbb{R}^{n_{1} \times n_{1}}$ and matrices $E_{v \mu} \in \mathbb{R}^{n_{1} \times n_{1}}$ for all $v, \mu \in\{1, \ldots, N\}$.

1. Let $Q_{v}$ be positive diagonal matrices for all $v \in\{1, \ldots, N\}$, and $A_{v \mu}$ be nonnegative for all $\mu, v \in\{1, \ldots, N\}$. Then, (A2) holds and the facts (F2) and (F3) are true.

2. Let $E_{\nu \mu}=0$ for all $\mu, v \in\{1, \ldots, N\}$. Then, $\tilde{M}$ is a $P_{0}$-matrix, and the facts (F6) and (F7) are true. If, moreover, $Q_{v}$ is positive definite for all $v=1, \ldots, N$, (A1) holds and facts (F1)-(F4), (F6) and (F7) are true.

Proof Point 1 is due to Proposition 5.1.

Let us consider point 2. Let arbitrary $\lambda, w \in \mathbb{R}_{++}^{3 n}$ be given. By assumption $J_{x} F$ is positive semidefinite. We set

$\widetilde{J_{x} L}(\lambda, w)=J_{x} F+\operatorname{diag}\left(\lambda^{2} \circ\left(w^{2}\right)^{-1}\right)+\operatorname{diag}\left(\lambda^{3} \circ\left(w^{3}\right)^{-1}\right)=:\left(\begin{array}{ccc}\widetilde{Q}_{1} & & \\ & \ddots & \\ & & \widetilde{Q}_{N}\end{array}\right)$,

with positive definite diagonal matrices $\widetilde{Q}_{v} \in \mathbb{R}^{n_{1} \times n_{1}}, v=1, \ldots, N$. We get

$$
\begin{aligned}
\tilde{M} & =\left(\begin{array}{ccc}
D_{1} \bar{D}_{1} & \cdots & D_{1} \bar{D}_{N} \\
\vdots & & \vdots \\
D_{N} \bar{D}_{1} & \cdots & D_{N} \bar{D}_{N}
\end{array}\right)\left(\begin{array}{ccc}
\widetilde{Q}_{1}^{-1} & & \\
& \ddots & \\
& & \widetilde{Q}_{N}^{-1}
\end{array}\right)\left(\begin{array}{ccc}
D_{1} \bar{D}_{1} & & \\
& \ddots & \\
& & D_{N} \bar{D}_{N}
\end{array}\right) \\
& =\left(\begin{array}{cccc}
D_{1} \bar{D}_{1} \widetilde{Q}_{1}^{-1} D_{1} \bar{D}_{1} & \cdots & D_{1} \bar{D}_{N} \widetilde{Q}_{N}^{-1} D_{N} \bar{D}_{N} \\
\vdots & & & \\
& & & \\
D_{N} \bar{D}_{1} \widetilde{Q}_{1}^{-1} D_{1} \bar{D}_{1} & \cdots & D_{N} \bar{D}_{N} \widetilde{Q}_{N}^{-1} D_{N} \bar{D}_{N}
\end{array}\right)
\end{aligned}
$$




$$
=C C^{\top} \check{D}
$$

where $C:=\left(\begin{array}{c}D_{1} \\ \vdots \\ D_{N}\end{array}\right)$ and $\check{D}:=\left(\begin{array}{ccc}\bar{D}_{1} \widetilde{Q}_{1}^{-1} \bar{D}_{1} & & \\ & \ddots & \\ & & \bar{D}_{N} \widetilde{Q}_{N}^{-1} \bar{D}_{N}\end{array}\right)$.

For arbitrary $J \subseteq\{1, \ldots, m\}$, we have

$$
\operatorname{det}\left(\widetilde{M}_{J J}\right)=\operatorname{det}\left(\left(C C^{\top}\right)_{J J} \check{D}_{J J}\right)=\operatorname{det}\left(\left(C C^{\top}\right)_{J J}\right) \operatorname{det}\left(\check{D}_{J J}\right) \geq 0
$$

because $C C^{\top}$ is positive semidefinite, and $\check{D}$ is positive semidefinite and diagonal. Therefore, $\tilde{M}$ is a $P_{0}$ matrix. Facts (F6) and (F7) follow from Corollary 5.1.

Moreover, if matrices $Q_{v}$ are positive definite, then we can exploit the point (c) of Corollary 5.2 to say that assumption (A1) holds. Then, the assertion can be obtained as in point 2 of Lemma 6.1.

Remark 6.1 Let us give an example class, where Lemma 6.2 can be applied for GNEPs. Suppose, each player has one variable, and, beside box constraints, we have exactly one constraint, that is shared by all players. Then, this constraint can be stated using $A_{\nu \mu}=\bar{D}_{\mu} \in \mathbb{R}$ and $D_{\nu}=1$ for all $\nu, \mu=1, \ldots, N$. Now, if all $Q_{\nu} \in \mathbb{R}_{++}$, the second case of Lemma 6.2 guarantees (F1)-(F4), (F6) and (F7).

Remark 6.2 Unfortunately, if we have GNEPs with two or more linearly independent shared constraints, the matrix $\widetilde{M}$ can easily fail to be a $P_{0}$-matrix. For example, consider a 2 player game, where each player has one variable and we have, beside box constraints, two shared constraints $a_{1} x^{1}+a_{2} x^{2} \leq b_{1}, a_{3} x^{1}+a_{4} x^{2} \leq b_{2}$. Then, if $\tilde{Q}_{1}, \tilde{Q}_{2}>0$ we have that $\widetilde{J_{x} L}=\left(\begin{array}{cc}\tilde{Q}_{1} & 0 \\ 0 & \tilde{Q}_{2}\end{array}\right)$ is nonsingular. Further,

$$
\tilde{M}=\left(\begin{array}{cccc}
a_{1}^{2} \tilde{Q}_{1}^{-1} & a_{1} a_{3} \tilde{Q}_{1}^{-1} & a_{2}^{2} \tilde{Q}_{2}^{-1} & a_{2} a_{4} \tilde{Q}_{2}^{-1} \\
a_{1} a_{3} \tilde{Q}_{1}^{-1} & a_{3}^{2} \tilde{Q}_{1}^{-1} & a_{2} a_{4} \tilde{Q}_{2}^{-1} & a_{4}^{2} \tilde{Q}_{2}^{-1} \\
a_{1}^{2} \tilde{Q}_{1}^{-1} & a_{1} a_{3} \tilde{Q}_{1}^{-1} & a_{2}^{2} \tilde{Q}_{2}^{-1} & a_{2} a_{4} \tilde{Q}_{2}^{-1} \\
a_{1} a_{3} \tilde{Q}_{1}^{-1} & a_{3}^{2} \tilde{Q}_{1}^{-1} & a_{2} a_{4} \tilde{Q}_{2}^{-1} & a_{4}^{2} \tilde{Q}_{2}^{-1}
\end{array}\right)
$$

For the index sets $I=\{2,3\}$ and $J=\{1,4\}$, we obtain

$$
\begin{aligned}
\operatorname{det}\left(\tilde{M}_{I I}\right) & =-a_{2} a_{3}\left(a_{1} a_{4}-a_{2} a_{3}\right) \tilde{Q}_{1}^{-1} \tilde{Q}_{2}^{-1}, \\
\operatorname{det}\left(\tilde{M}_{J J}\right) & =a_{1} a_{4}\left(a_{1} a_{4}-a_{2} a_{3}\right) \tilde{Q}_{1}^{-1} \tilde{Q}_{2}^{-1} .
\end{aligned}
$$

Now we see that $\tilde{M}$ is not a $P_{0}$ matrix, whenever $\operatorname{sign}\left(a_{1} a_{4}\right)=\operatorname{sign}\left(a_{2} a_{3}\right) \neq 0$. In this case, by point (c) of Corollary 5.2 we obtain that the matrix $M$ is not a $P_{0}$ matrix. Then, Corollary 4.1 shows that $J H(x, \lambda, w)$ fails to be nonsingular for all $(\lambda, w) \in \mathbb{R}_{++}^{2} \times \mathbb{R}_{++}^{2}$.

Finally, we want to present some GNEPs with box constraints, where (F5) can be shown. We discuss an example class that often occurs in applications. 
Proposition 6.1 Let $X_{v}=\left[l^{v}, u^{v}\right]$ for all $v=1, \ldots, N$, and further, only upper constraints are present, i.e., all coefficients of the remaining constraints are positive. Let the feasible set be nonempty. If all $Q_{v} \in \mathbb{R}^{n_{\nu} \times n_{\nu}}, v=1, \ldots, N$ are nonsingular and $M$ is positive semidefinite, (F5) holds.

Proof Since all $Q_{\nu} \in \mathbb{R}^{n_{\nu} \times n_{\nu}}, v=1, \ldots, N$ are nonsingular, $J_{x} L=J_{x} F$ is nonsingular. Since $M$ is positive semidefinite by assumption, we can apply Theorem 5.1, if we have shown that the columns of the matrix $\nabla h_{\bullet J}(x)$ with $J=\left\{j \in\{1, \ldots, m\}: h_{j}(x)>0\right\}$ are positively linearly independent for all $x \in \mathbb{R}^{n}$. Assume we have a vector $v \in \mathbb{R}_{+}^{|J|}$ with $\nabla h_{\bullet J}(x) v=0$. We want to show that $v=0$ must hold.

Whenever $J$ contains an upper box constraint with index $j_{0}$, the corresponding lower box constraint is not in $J$. Hence, the matrix $\nabla h_{\bullet} J(x)$ has a corresponding row, with only nonnegative entries, and we must have $v_{j_{0}}=0$. Thus, we can assume without loss of generality that $J$ does not contain any upper box constraint.

Since the feasible set is nonempty, the set $J=\left\{j \in\{1, \ldots, m\}: h_{j}(x)>0\right\}$ cannot contain all lower box constraints and an additional upper constraint. Hence, the matrix $\nabla h_{\bullet J}(x)$ can either contain only lower box constraints, resulting in $v=0$, or it must contain at least one row where all entries are nonnegative. But then, the components of $v$ corresponding to the positive coefficients of the upper constraints must all be zero. The remaining matrix has only lower box constraints, again resulting in $v=0$. Thus, we must have $v=0$, meaning that the columns of $\nabla h_{\bullet J}(x)$ are positively linear independent for all $x \in \mathbb{R}^{n}$. By Theorem 5.1, fact (F5) holds.

\section{Conclusions}

In this paper, we presented a number of necessary and sufficient conditions for the main issues, when solving QVIs via a smooth constrained or a nonsmooth unconstrained equation reformulation of their KKT conditions. We have seen that the matrix $M$ plays a crucial role. The first issue is to guarantee that stationary points of the merit function are indeed solutions. We considered several concepts of constrained or unconstrained stationary points, and the issue was solved under a $P_{0}$ property for the nonsmooth reformulation. For the constrained, but smooth, reformulation we required stronger assumptions, namely positive semidefiniteness of $M$ together with the $S$ property of this matrix. Further, we gave an example, showing that the $P_{0}$ property is not sufficient in the smooth reformulation. Considering only stationary points within the feasible set, which is important for interior point methods, we showed the stationarity result for both reformulations, if $M$ is a semimonotone matrix.

For the second issue, the nonsingularity of the Jacobian at feasible iterates, we considered the QVI class, where the constraints are linear with variable right-hand side. Here, we proved equivalence of the nonsingularity with a sign condition for determinants of certain matrices, which are independent of the dual variables. Furthermore, under an additional nonsingularity assumption on $J_{x} F(x)$, we showed equivalence to the $P_{0}$ property of $M$. In the context of QVIs, these are, to the best of our knowledge, the first necessary and sufficient conditions for nonsingularity of the Jacobians. We 
have also seen that, for QVIs with box constraints, some of the assumptions can be weakened.

We further presented some subclasses of GNEPs with linear constraints and quadratic cost functions, for which the Jacobian is always nonsingular, and the absence of nonoptimal stationary points can be shown. Moreover, we extended a known sufficient nonsingularity condition for GNEPs with linear constraints and cost functions to a necessary and sufficient condition.

Acknowledgements Open Access funding provided by Projekt DEAL.

\section{Compliance with ethical standards}

Conflict of interest The authors declare that they have no conflict of interest.

Open Access This article is licensed under a Creative Commons Attribution 4.0 International License, which permits use, sharing, adaptation, distribution and reproduction in any medium or format, as long as you give appropriate credit to the original author(s) and the source, provide a link to the Creative Commons licence, and indicate if changes were made. The images or other third party material in this article are included in the article's Creative Commons licence, unless indicated otherwise in a credit line to the material. If material is not included in the article's Creative Commons licence and your intended use is not permitted by statutory regulation or exceeds the permitted use, you will need to obtain permission directly from the copyright holder. To view a copy of this licence, visit http://creativecommons.org/licenses/by/4.0/.

\section{References}

1. Bensoussan, A., Goursat, M., Lions, J.L.: Contrôle impulsionnel et inéquations quasi-variationnelles stationnaires. CR Acad. Sci. Paris Sér. A 276, 1279-1284 (1973)

2. Bensoussan, A., Lions, J.L.: Nouvelle formulation de problèmes de contrôle impulsionnel et applications. CR Acad. Sci. Paris Sér. A 276, 1189-1192 (1973)

3. Bensoussan, A., Lions, J.L.: Nouvelles methodes en contrôle impulsionnel. Appl. Math. Optim. 1(4), 289-312 (1975)

4. Baiocchi, C., Capelo, A.: Variational and Quasivariational Inequalities: Applications to Free Boundary Problems. Wiley, New York (1984)

5. Mosco, U.: Implicit variational problems and quasi variational inequalities. In: Gossez, J.P., Lami Dozo, E.J., Mawhin, J., Waelbroeck, L. (eds.) Nonlinear Operators and the Calculus of Variations, pp. 83-156. Springer, Berlin (1976)

6. Debreu, G.: A social equilibrium existence theorem. Proc. Natl. Acad. Sci. USA 38(10), 886-893 (1952)

7. Bensoussan, A.: Points de Nash dans le cas de fonctionnelles quadratiques et jeux differentiels lineaires a N personnes. SIAM J. Control 12(3), 460-499 (1974)

8. Facchinei, F., Kanzow, C.: Penalty methods for the solution of generalized nash equilibrium problems. SIAM J. Optim. 20, 2228-2253 (2010)

9. Hammerstein, P., Selten, R.: Game theory and evolutionary biology. In: Aumann, R.J., Hart, S. (eds.) Handbook of Game Theory with Economic Applications, vol. 2, pp. 929-993. Elsevier, North-Holland, Amsterdam (1994)

10. Ichiishi, T.: Game Theory for Economic Analysis. Academic Press, New York (1983)

11. Jing-Yuan, W., Smeers, Y.: Spatial oligopolistic electricity models with cournot generators and regulated transmission prices. Oper. Res. 47(1), 102-112 (1999)

12. Bliemer, M.C., Bovy, P.H.: Quasi-variational inequality formulation of the multiclass dynamic traffic assignment problem. Transp. Res. Part B Methodol. 37(6), 501-519 (2003)

13. Scrimali, L.: Quasi-variational inequalities in transportation networks. Math. Models Methods Appl. Sci. 14(10), 1541-1560 (2004) 
14. Dreves, A.: Uniqueness for quasi-variational inequalities. Set Valued Var. Anal. 24(2), 285-297 (2016)

15. Nesterov, Y., Scrimali, L.: Solving strongly monotone variational and quasi-variational inequalities. CORE Discussion Papers 2006107, Université catholique de Louvain, Center for Operations Research and Econometrics (CORE) (2006)

16. Dreves, A., Facchinei, F., Fischer, A., Herrich, M.: A new error bound result for generalized Nash equilibrium problems and its algorithmic application. Comput. Optim. Appl. 59(1), 63-84 (2014)

17. Izmailov, A.F., Solodov, M.V.: On error bounds and Newton-type methods for generalized Nash equilibrium problems. Comput. Optim. Appl. 59(1), 201-218 (2014)

18. Facchinei, F., Kanzow, C., Karl, S., Sagratella, S.: The semismooth Newton method for the solution of quasi-variational inequalities. Comput. Optim. Appl. 62(1), 85-109 (2015)

19. Dreves, A., Facchinei, F., Kanzow, C., Sagratella, S.: On the solution of the KKT conditions of generalized Nash equilibrium problems. SIAM J. Optim. 21(3), 1082-1108 (2011)

20. Yamashita, N., Fukushima, M.: On the rate of convergence of the Levenberg-Marquardt method. In: Alefeld, G., Chen, X. (eds.) Topics in Numerical Analysis, pp. 239-249. Springer, Vienna (2001)

21. Facchinei, F., Kanzow, C., Sagratella, S.: Solving quasi-variational inequalities via their KKT conditions. Math. Program. 144(1), 369-412 (2014)

22. Bellavia, S., Macconi, M., Morini, B.: An affine scaling trust-region approach to bound-constrained nonlinear systems. Appl. Numer. Math. 44(3), 257-280 (2003)

23. Bellavia, S., Macconi, M., Morini, B.: STRSCNE: a scaled trust-region solver for constrained nonlinear equations. Comput. Optim. Appl. 28(1), 31-50 (2004)

24. Galli, L., Kanzow, C., Sciandrone, M.: A nonmonotone trust-region method for generalized Nash equilibrium and related problems with strong convergence properties. Comput. Optim. Appl. 69(3), 629-652 (2018)

25. Facchinei, F., Fischer, A., Herrich, M.: An LP-Newton method: nonsmooth equations, KKT systems, and nonisolated solutions. Math. Program. 146(1), 1-36 (2014)

26. Fischer, A., Herrich, M., Izmailov, A., Solodov, M.: A globally convergent LP-Newton method. SIAM J. Optim. 26(4), 2012-2033 (2016)

27. Aussel, D., Sagratella, S.: Sufficient conditions to compute any solution of a quasivariational inequality via a variational inequality. Math. Methods Oper. Res. 85(1), 3-18 (2017)

28. Latorre, V., Sagratella, S.: A canonical duality approach for the solution of affine quasi-variational inequalities. J. Global Optim. 64(3), 433-449 (2016)

29. Kanzow, C.: On the multiplier-penalty-approach for quasi-variational inequalities. Math. Program. 160(1), 33-63 (2016)

30. Kanzow, C., Steck, D.: Augmented Lagrangian and exact penalty methods for quasi-variational inequalities. Comput. Optim. Appl. 69(3), 801-824 (2018)

31. Pang, J.S., Fukushima, M.: Quasi-variational inequalities, generalized Nash equilibria, and multileader-follower games. Comput. Manag. Sci. 2(1), 21-56 (2005)

32. Dreves, A., Sudermann-Merx, N.: Solving linear generalized Nash equilibrium problems numerically. Optim. Methods Softw. 31(5), 1036-1063 (2016)

33. Cottle, R., Pang, J.S., Stone, R.: The Linear Complementarity Problem. Academic Press, New York (1992)

34. Facchinei, F., Fischer, A., Kanzow, C., Peng, J.M.: A simply constrained optimization reformulation of KKT systems arising from variational inequalities. Appl. Math. Optim. 40(1), 19-37 (1999)

35. Sandberg, I.W., Willson, A.N.: Some theorems on properties of DC equations of nonlinear networks. Bell Syst. Tech. J. 48(1), 1-34 (1969)

36. Mangasarian, O.: Nonlinear Programming. McGraw-Hill, New York (1969)

37. Facchinei, F., Piccialli, V., Sciandrone, M.: Decomposition algorithms for generalized potential games. Comput. Optim. Appl. 50(2), 237-262 (2011)

38. Sagratella, S.: Algorithms for generalized potential games with mixed-integer variables. Comput. Optim. Appl. 68(3), 689-717 (2017)

Publisher's Note Springer Nature remains neutral with regard to jurisdictional claims in published maps and institutional affiliations. 